\title{
A Unified Theory (I) for Neighborhood Systems and Basic Concepts on Fuzzifying Topological Spaces
}

\author{
Osama Rashed Sayed \\ Department of Mathematics, Faculty of Science, Assiut University, Assiut, Egypt \\ Email: o_r_sayed@yahoo.com
}

Received September 10, 2011; revised August 7, 2012; accepted August 14, 2012

\begin{abstract}
This paper considers fuzzifying topologies, a special case of I-fuzzy topologies (bifuzzy topologies), introduced by Ying [25]. It investigates topological notions defined by means of $\Delta$-open sets when these are planted into the framework of Ying's fuzzifying topological spaces (by Łukasiewicz logic in $[0,1])$. In this paper we introduce some sorts of operations, called general fuzzifying operations from $P(X)$ to $\Im(X)$, where $(X, \tau)$ is a fuzzifying topological space. By making use of them we contract neighborhood structures, derived sets, closure operations and interior operations.
\end{abstract}

Keywords: Łukasiewicz Logic; Semantics; Fuzzifying Topology

\section{Introduction}

In the last few years fuzzy topology, as an important research field in fuzzy set theory, has been developed into a quite mature discipline [1-6]. In contrast to classical topology, fuzzy topology is endowed with richer structure, to a certain extent, which is manifested with different ways to generalize certain classical concepts. So far, according to Ref. [2], the kind of topologies defined by Chang [7] and Goguen [8] is called the topologies of fuzzy subsets, and further is naturally called L-topological spaces if a lattice $L$ of membership values has been chosen. Loosely speaking, a topology of fuzzy subsets (resp. an L-topological space) is a family $\tau$ of fuzzy subsets (resp. L-fuzzy subsets) of nonempty set $X$, and $\tau$ satisfies the basic conditions of classical topologies [9]. On the other hand, Höhle in [10] proposed the terminology L-fuzzy topology to be an L-valued mapping on the traditional powerset $P(X)$ of $X$. The authors in $[4,5,11,12]$ defined an L-fuzzy topology to be an L-valued mapping on the L-powerset $L^{X}$ of $X$. In 1952, Rosser and Turquette [13] proposed emphatically the following problem: If there are many-valued theories beyond the level of predicates calculus, then what are the detail of such theories? As an attempt to give a partial answer to this problem in the case of point set topology, Ying in $1991[14,15]$ used a semantical method of continuousvalued logic to develop systematically fuzzifying topology. Briefly speaking, a fuzzifying topology on a set $X$ assigns each crisp subset of $X$ to a certain degree of being open, other than being definitely open or not. In fact, fuzzifying topologies are a special case of the L-fuzzy topologies in $[11,12]$ since all the t-norms on I are included as a special class of tensor products in these paper. Ying uses one particular tensor product, namely Łukasiewicz conjunction. Thus his fuzzifying topologies are a special class of all the I-fuzzy topologies considered in the categorical frameworks [11,12]. Roughly speaking, the semantical analysis approach transforms formal statements of interest, which are usually expressed as implication formulas in logical language, into some inequalities in the truth value set by truth valuation rules, and then these inequalities are demonstrated in an algebraic way and the semantic validity of conclusions is thus established. So far, there has been significant research on fuzzifying topologies [16-21]. In 1979, several characterizations of compactness are unified by the operation introduced by Kasahara [22]. Also, he studied the concept of $\alpha$-continuity (where $\alpha$ is an operation) and defined some types of spaces by using this operation. In 1981, the concept of other type of continuity which generalizes the $\alpha$-continuity [22] was introduced by Jankovic [23]. In 1983, Abd El-Monsef, et al. [24] introduced an operation $\tilde{\alpha}$ on the family $\mathfrak{J}$ of all closed sets in the topological space $(X, \tau)$ which is dual to the operation $\alpha$. In 1991, Kerre et al. [25] introduced an extension of the concept of an operation on the class of all fuzzy sets on $X$ endowed with Chang fuzzy topology [7]. It was shown that a lot of characterizations and properties of many concepts and stronger forms can be unified by using this notion. In 1991, Kandil et al. [26] applied the 
concept of the operation defined in [25] to unify and generalize several characterizations and properties of a lot of already existing weaker and stronger forms of fuzzy continuity. A basic structure of this paper is as follows: First, in Section 2 we offer some definition and results which will be needed in this paper. In Section 3 the concepts of fuzzifying $\Delta$-open sets, $C \Delta$-open sets, $\Delta$-closed sets and $C \Delta$-closed sets are introduced and some of their properties are discussed. In Section 4 the fuzzifying $\Delta$ - and $C \Delta$-neighborhood systems are presented and a fuzzifying topology induced by $C \Delta$ neighborhood system is introduced. In Section 5 the concepts of fuzzifying $\Delta$ - and $C \Delta$-derived sets, $\Delta$ - and $C \Delta$-closure operations and $\Delta$ - and $C \Delta$-interior operations were established and some of their properties are studied. Finally, in Section 6, we summarize the main results obtained and raise some related problems for further study. Thus we fill a gap in the existing literature on fuzzifying topology.

Note: All corollaries in this paper are results in [1421].

\section{Preliminaries}

We present the fuzzy logical and corresponding set theoretical notations $[14,15]$ since we need them in this paper.

For any formula $\varphi$, the symbol $[\varphi]$ means the truth value of $\varphi$, where the set of truth values is the unit interval $[0,1]$. We write $\vDash \varphi$ if $[\varphi]=1$ for any interpretation. Also, $\mathfrak{I}(X)$ is the family of all fuzzy sets in $X$. The truth valuation rules for primary fuzzy logical formulae and corresponding set theoretical notations are:

1) a) $[\alpha]=\alpha(\alpha \in[0,1])$;

b) $[\varphi \wedge \psi]=\min ([\varphi],[\psi])$;

c) $[\varphi \rightarrow \psi]=\min (1,1-[\varphi]+[\psi])$.

2) If $\tilde{A} \in \mathfrak{I}(X),[x \in \tilde{A}]:=\tilde{A}(x)$.

3) If $X$ is the universe of discourse, then

$$
[\forall x \varphi(x)]:=\inf _{x \in X}[\varphi(x)] .
$$

In addition the truth valuation rules for derived formulae are:

1) $[\neg \varphi]:=[\varphi \rightarrow 0]=1-[\varphi]$;

2) $[\varphi \vee \psi]:=[\neg(\neg \varphi \wedge \neg \psi)]=\max ([\varphi],[\psi])$;

3) $[\varphi \leftrightarrow \psi]:=[(\varphi \rightarrow \psi) \wedge(\psi \rightarrow \varphi)]$;

4) $[\varphi \otimes \psi]:=[\neg(\varphi \rightarrow \neg \psi)]=\max (0,[\varphi]+[\psi]-1)$;

5) $[\exists x \varphi(x)]:=[\neg \forall x \neg \varphi(x)]:=\sup _{x \in X}[\varphi(x)]$;

6) If $\tilde{A}, \tilde{B} \in \mathfrak{J}(X)$, then a) $[\tilde{A} \subseteq \tilde{B}]:=[\forall x(x \in \tilde{A} \rightarrow x \in \tilde{B})]$

$$
=\inf _{x \in X} \min (1,1-\tilde{A}(x)+\tilde{B}(x)) ;
$$

b) $[\tilde{A} \equiv \tilde{B}]:=[\tilde{A} \subseteq \tilde{B}] \wedge[\tilde{B} \subseteq \tilde{A}]$;

c) $[\tilde{A} \doteq \tilde{B}]:=[\tilde{A} \subseteq \tilde{B}] \otimes[\tilde{B} \subseteq \tilde{A}]$.

We give now the following definitions and results in fuzzifying topology [14-21] which are used in the sequel.

Definition 2.1 [14]. Let $X$ be a universe of discourse, and $\tau \in \mathfrak{I}(P(X))$ satisfy the following conditions:

1) $\tau(X)=\tau(\varnothing)=1$;

2) for any $A, B \in P(X), \tau(A \cap B) \geq \tau(A) \wedge \tau(B)$;

3) for any

$$
\left\{A_{\lambda} \in P(X): \lambda \in \Lambda\right\}, \tau\left(\bigcup_{\lambda \in \Lambda} A_{\lambda}\right) \geq \underset{\lambda \in \Lambda}{\wedge} \tau\left(A_{\lambda}\right) .
$$

Then $\tau$ is a fuzzifying topology and $(X, \tau)$ is a fuzzifying topological space.

Note: In the rest of this paper $(X, \tau)$ (or briefly $X)$ is always fuzzifying topological space.

Definition 2.2 [14] The family of all fuzzifying closed sets, denoted by $F \in \mathfrak{I}(P(X))$, is defined as $A \in F:=(X-A) \in \tau$, where $X-A$ is the complement of $A$.

Definition 2.3 [14] The neighborhood system $N_{x} \in \mathfrak{I}(P(X))$ of $x \in X$ is defined as

$$
N_{x}(A)=\sup _{x \in B \subseteq A} \tau(B) .
$$

Definition 2.4 [15] The interior $A^{\circ}$ or $\operatorname{Int}(A)$ of $A \subseteq X$ is defined as $\operatorname{Int}(A)(x)=N_{x}(A)$.

Definition 2.5 (Lemma 5.2. [14]). The closure $\mathrm{Cl}(A)$ or $\bar{A}$ of $A$ is defined as $C l(A)(x)=1-N_{x}(X-A)$. In Theorem 5.3 [14], Ying proved that the closure $C l: P(X) \rightarrow \mathfrak{I}(X)$ is a fuzzifying closure operator since

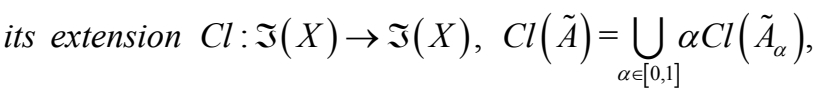
$\tilde{A} \in \mathfrak{I}(X)$, where $\tilde{A}_{\alpha}=\{\alpha: \tilde{A}(x) \geq \alpha\}$ is the $\alpha$-cut of $A$ and $\alpha \tilde{A}=\alpha \wedge \tilde{A}(x)$ satisfies the following Kuratowski closure axioms:

1) $\vDash C l(\varnothing)=\varnothing$;

2) for any $\tilde{A} \in \mathfrak{I}(X), \quad \vDash \tilde{A} \subseteq C l(\tilde{A})$;

3) for any

$$
\tilde{A}, \tilde{B} \in \mathfrak{I}(X), \models C l(\tilde{A} \cup \tilde{B})=C l(\tilde{A}) \cup C l(\tilde{B}) ;
$$

4) for any $\tilde{A} \in \mathfrak{J}(X), \models C l(C l(\tilde{A})) \subseteq C l(\tilde{A})$.

Definition 2.6 [18] For any $\tilde{A} \in \mathfrak{I}(X)$, $\vDash \operatorname{Int}(\tilde{A}) \equiv X-C l(X-\tilde{A})$.

Theorem 2.1 [18] For any $\tilde{A}, \tilde{B} \in \mathfrak{I}(X)$, 
1) $\vDash \operatorname{Int}(X) \equiv X$;

2) $\vDash \operatorname{Int}(\tilde{A}) \subseteq \tilde{A}$;

3) $\vDash \operatorname{Int}(\tilde{A} \cap \tilde{B})=\operatorname{Int}(\tilde{A}) \cap \operatorname{Int}(\tilde{B})$;

4) $\vDash \operatorname{Int}(\operatorname{Int}(\tilde{A})) \supseteq \operatorname{Int}(\tilde{A})$.

Theorem 2.2 [18] For any $\tilde{A}, \tilde{B} \in \mathfrak{I}(X)$, if $[\tilde{A} \subseteq \tilde{B}]=1$, then

1) $\vDash C l(\tilde{A}) \subseteq C l(\tilde{B})$;

2) $\vDash \operatorname{Int}(\tilde{A}) \subseteq \operatorname{Int}(\tilde{B})$;

3) $\vDash \operatorname{Int}(\operatorname{Cl}(\operatorname{Int}(\tilde{A}))) \subseteq \operatorname{Int}(\operatorname{Cl}(\operatorname{Int}(\tilde{B})))$;

4) $\vDash C l(\operatorname{Int}(\tilde{A})) \subseteq C l(\operatorname{Int}(\tilde{B}))$;

5) $\vDash \operatorname{Int}(C l(\tilde{A})) \subseteq \operatorname{Int}(C l(\tilde{B}))$;

6) $\vDash C l(\operatorname{Int}(C l(\tilde{A}))) \subseteq C l(\operatorname{Int}(C l(\tilde{B})))$.

Theorem 2.3 For any $\tilde{A} \in \mathfrak{J}(X)$,

1) $\vDash X-\operatorname{Int}(C l(\operatorname{Int}(\tilde{A}))) \equiv C l(\operatorname{Int}(C l(X-\tilde{A})))$ $[19]$

2) $\vDash X-C l(\operatorname{Int}(\tilde{A})) \equiv \operatorname{Int}(C l(X-\tilde{A}))[18]$;

3) $\vDash X-C l(\operatorname{Int}(\tilde{A})) \equiv C l(\operatorname{Int}(X-\tilde{A})) \quad[18]$;

4) $\vDash X-C l(\operatorname{Int}(C l(\tilde{A}))) \equiv \operatorname{Int}(C l(\operatorname{Int}(X-\tilde{A})))[16]$.

Theorem 2.4

1) $\vDash X \equiv \operatorname{Int}(C l(\operatorname{Int}(X))) ; \vDash \phi \equiv \operatorname{Int}(C l(\operatorname{Int}(\phi)))$;

2) $\vDash X \equiv C l(\operatorname{Int}(X)) ; \vDash \phi \equiv C l(\operatorname{Int}(\phi))$;

3) $\vDash X \equiv \operatorname{Int}(C l(X)) ; \vDash \phi \equiv \operatorname{Int}(C l(\phi))$;

4) $\vDash X \equiv C l(\operatorname{Int}(C l(X))) ; \vDash \phi \equiv C l(\operatorname{Int}(C l(\phi)))$.

Theorem 2.5 For any $A \in P(X)$,

1) $\vDash \operatorname{Int}(A) \subseteq \operatorname{Int}(C l(\operatorname{Int}(A)))[19]$;

2) $\vDash \operatorname{Int}(C l(\operatorname{Int}(A))) \subseteq \operatorname{Int}(C l(A))$;

3) $\vDash \operatorname{Int}(C l(\operatorname{Int}(A))) \subseteq C l(\operatorname{Int}(A))$;

4) $\vDash C l(\operatorname{Int}(A)) \subseteq C l(\operatorname{Int}(C l(A)))$;

5) $\vDash \operatorname{Int}(C l(A)) \subseteq C l(\operatorname{Int}(C l(A)))$.

Theorem 2.6 For any $A \in P(X)$,

1) $\vDash \operatorname{Int}(A) \subseteq C l(\operatorname{Int}(A))$;

2) $\vDash \operatorname{Int}(A) \subseteq \operatorname{Int}(C l(A)) \quad[17]$;

3) $\vDash \operatorname{Int}(A) \subseteq C l(\operatorname{Int}(C l(A))) \quad[16]$.

Definition 2.7 Let $X$ be a non-empty set.
1) By the symbol $\mathfrak{I}(X)^{P(X)}$ we denote the set of all functions from $P(X)$ into $\mathfrak{I}(X)$. Each member of $\mathfrak{J}(X)^{P(X)}$ will be called a general fuzzifying operation.

2) Let $\Delta, \nabla \in \mathfrak{I}(X)^{P(X)}$.

a) We say that $\Delta \leq \nabla$, if $\Delta(A) \leq \nabla(A)$ for each $A \subseteq X$.

b) We say that $\Delta$ and $\nabla$ are dual if $\vDash X-\Delta(A) \equiv \nabla(X-A)$; equivalently $\vDash X-\nabla(A) \equiv \Delta(X-A)$ for each $A \subseteq X$.

3) A general fuzzifying operation $\Delta \in \mathfrak{J}(X)^{P(X)}$ is said to be monotone if $\vDash A \subseteq B \rightarrow \Delta(A) \subseteq \Delta(B)$.

4) A general fuzzifying operation $\Delta \in \mathfrak{J}(X)^{P(X)}$ is said to be of type $O 1$ if $\vDash X \subseteq \Delta(X)$; equivalently $\vDash \nabla(\phi) \subseteq \phi$, where $\Delta$ and $\nabla$ are dual.

5) A general fuzzifying operation $\Delta \in \mathfrak{I}(X)^{P(X)}$ is said to be of type $O 2$ if $\Delta(A \cap B)=\Delta(A) \wedge \Delta(B)$ for any $A, B \subseteq X$.

Example 2.1 1) From Theorem 2.4 we have ()$^{\circ}$, ()$^{-},()^{\circ-\circ},()^{-\circ},()^{\circ-}, 0^{-\circ} \cup()^{\circ-}$, and $\left(^{-\circ-}\right.$ are of type $\mathrm{O} 1$ and each member of them is monotone from Theorem 2.2.

2) The fuzzifying operations ()$^{\circ},()^{-},()^{\circ-\circ}, 0^{-\circ},()^{\circ-}$, ()$^{-\circ} \cup()^{\circ-}$, and ()$^{-\circ-}$ and the fuzzifying operations $0^{-},\left(0^{\circ},()^{-\circ-},()^{\circ-},()^{-\circ}, 0^{-\circ} \cup()^{\circ-}\right.$, and $0^{\circ-\circ}$ are dual respectively (see Theorem 2.3).

3) From Theorem 2.1 (3), $)^{\circ}$ is of type $O 2$.

4) From Theorem 2.5 , one can easily deduce that:

a) ()$^{\circ} \leq()^{\circ-\circ}$;

b) ()$^{\circ-\circ} \leq()^{-\circ}$;

c) ()$^{\circ-\circ} \leq()^{\circ-}$;

d) $0^{\circ-\circ} \leq 0^{-\circ} \cup\left(0^{\circ-}\right.$;

e) ()$^{\circ-} \leq()^{-\circ-}$;

f) ()$^{-\circ} \leq()^{-\circ-}$;

g) ()$^{-\circ} \cup()^{\circ-} \leq()^{-\circ-}$.

Note: In the rest of this paper always $\Delta, \nabla \in \mathfrak{I}(X)^{P(X)}$.

\section{Fuzzifying Open Sets}

In this section the concepts of fuzzifying $\Delta$-open sets, $C \Delta$-open sets, $\Delta$-closed sets and $C \Delta$-closed sets are introduced and some of their properties are discussed.

Definition 3.1 1) The family of all fuzzifying $\Delta$-open sets, denoted by $\tau_{\Delta} \in \mathfrak{T}(P(X))$, is defined as follows:

$$
A \in \tau_{\Delta}:=\forall x(x \in A \rightarrow x \in \Delta(A)),
$$

i.e.,

$$
\tau_{\Delta}(A)=\inf _{x \in A} \Delta(A)(x) ;
$$

2) The family of all fuzzifying $C \Delta$-open sets, denoted by $\tau_{C \Delta} \in \mathfrak{I}(P(X))$, is defined as follows:

$$
A \in \tau_{C \Delta}:=\forall x\left(x \in A \cap \Delta(A) \rightarrow x \in A^{\circ}\right),
$$


i.e.,

$$
\tau_{C \Delta}(A)=\inf _{x \in A} \min \left(1,1-\Delta(A)(x)+A^{\circ}(x)\right) ;
$$

3) The family of all fuzzifying $\Delta$ (resp. $C \Delta$-closed sets, denoted by $\digamma_{\Delta}$ (resp. $\left.\digamma_{C \Delta}\right) \in \mathfrak{J}(P(X))$, is defined as follows:

$$
A \in \digamma_{\Delta}\left(\text { resp. } \digamma_{C \Delta}\right):=X-A \in \tau_{\Delta}\left(\operatorname{resp} . \tau_{C \Delta}\right)
$$

Definition 3.2 1) If $\Delta=()^{\circ}$, (resp. ()$^{\circ-\circ},()^{\circ-},()^{-\circ}$, ()$\left.^{-\circ} \cup()^{\circ-},()^{-\circ-}\right)$, the notion of fuzzifying $\Delta$-open sets coincides with the notion of fuzzifying open (resp. $\alpha$ open, semi-open, pre-open, $\gamma$-open, $\beta$-open) sets and will be denoted by $\tau$ (resp. $\left.\tau_{\alpha}, \tau_{s}, \tau_{p}, \tau_{\gamma}, \tau_{\beta}\right)$;

2) If $\Delta=()^{\circ-\circ}$ (resp. ()$^{\circ-},()^{-\circ},()^{-\circ} \cup()^{\circ-},()^{-\circ-}$ ), the notion of fuzzifying $C \Delta$-open sets coincides with the notion of fuzzifying $c \alpha$ (resp. csemi, cpre, $c \gamma, c \beta$ )open sets and will be denoted by $\tau_{C \alpha}$ (resp. $\tau_{C S}, \tau_{C P}$, $\left.\tau_{C \gamma}, \tau_{C \beta}\right)$;

3) If $\Delta=0^{\circ},\left(\right.$ resp. ()$\left.^{\circ-\circ},()^{\circ},()^{-\circ},()^{-\circ} \cup()^{\circ-},()^{-\circ-}\right)$, the notion of fuzzifying $\Delta$-closed sets coincides with the notion of fuzzifying closed (resp. $\alpha$-closed, semiclosed, pre-closed, $\gamma$-closed, $\beta$-closed) sets and will be denoted by $\digamma$ (resp. $\left.\digamma_{\alpha}, \digamma_{S}, \digamma_{P}, \digamma_{\gamma}, \digamma_{\beta}\right)$;

4) If $\Delta=()^{\circ-\circ}$ (resp. ()$\left.^{\circ-},()^{-\circ},()^{-\circ} \cup()^{\circ-},()^{-\circ-}\right)$, the notion of fuzzifying $C \Delta$-closed sets coincides with the notion of fuzzifying $c \alpha$ (resp. csemi, cpre, $c \gamma$, $c \beta$ )-closed sets and will be denoted by $\digamma_{C \alpha}$ (resp. $\left.\digamma_{C S}, \digamma_{C P}, \digamma_{C \gamma}, \digamma_{C \beta}\right)$;

Theorem 3.1 1) If $\Delta$ is of type O1, then

a) $\vDash X \in \tau_{\Delta} ; \vDash \phi \in \tau_{\Delta}$;

b) $\vDash X \in \tau_{C \Delta} ; \vDash \phi \in \tau_{C \Delta}$;

2) If $\Delta$ is monotone, then

a) for any $\left\{A_{\lambda}: \lambda \in \Lambda\right\} \subseteq P(X)$,

$$
\tau_{\Delta}\left(\bigcup_{\lambda \in \Lambda} A_{\lambda}\right) \geq{ }_{\lambda \in \Lambda} \tau_{\Delta}\left(A_{\lambda}\right) .
$$

b) for any $A, B \in P(X)$,

$$
\tau_{C \Delta}(A \cap B) \geq \tau_{C \Delta}(A) \wedge \tau_{C \Delta}(B)
$$

Proof. 1) a) $\tau_{\Delta}(X)=[X \subseteq \Delta(X)]=1$;

$\tau_{\Delta}(\phi)=[\phi \subseteq \Delta(\phi)]=1$.

b) $\tau_{C \Delta}(X)=\left[X \cap \Delta(X) \subseteq X^{\circ}\right]=[X \subseteq X]=1$;

$\tau_{C \Delta}(\phi)=\left[\phi \cap \Delta(\phi) \subseteq \phi^{\circ}\right]=[\phi \subseteq \phi]=1$.

2) a) Since $\Delta$ is monotone, then

$$
\begin{aligned}
\tau_{\Delta}\left(\bigcup_{\lambda \in \Lambda} A_{\lambda}\right) & =\inf _{x \in \bigcup_{\lambda \in \Lambda} A_{\lambda}} \Delta\left(\bigcup_{\lambda \in \Lambda} A_{\lambda}\right)(x) \\
& =\inf _{\lambda \in \Lambda} \inf _{x \in A_{\lambda}} \Delta\left(\bigcup_{\lambda \in \Lambda} A_{\lambda}\right)(x) \\
& \geq \inf _{\lambda \in \Lambda} \inf _{x \in A_{\lambda}} \Delta\left(A_{\lambda}\right)(x)={ }_{\lambda \in \Lambda} \tau_{\Delta}(A) .
\end{aligned}
$$

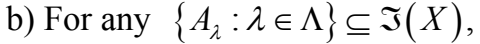

$$
\vDash \forall \lambda\left(\lambda \in \Lambda \rightarrow \tilde{A}_{\lambda} \subseteq \tilde{B}_{\lambda}\right) \rightarrow \bigcap_{\lambda \in \Lambda} \tilde{A}_{\lambda} \subseteq \bigcap_{\lambda \in \Lambda} \tilde{B}_{\lambda}
$$

(see Lemma 1.1 (1) [14]). Since $\Delta$ is monotone, then from Theorem 2.1 (3) we have

$$
\begin{aligned}
& \tau_{C \Delta}(A) \wedge \tau_{C \Delta}(B) \\
& =\left[A \cap \Delta(A) \subseteq A^{\circ} \wedge B \cap \Delta(B) \subseteq B^{\circ}\right] \\
& \leq\left[(A \cap B) \cap(\Delta(A) \cap \Delta(B)) \subseteq\left(A^{\circ} \cap B^{\circ}\right)\right] \\
& =\left[(A \cap B) \cap(\Delta(A) \cap \Delta(B)) \subseteq(A \cap B)^{\circ}\right] \\
& \leq\left[(A \cap B) \cap \Delta(A \cap B) \subseteq(A \cap B)^{\circ}\right] \\
& =\tau_{C \Delta}(A \cap B) .
\end{aligned}
$$

Corollary 3.1 1) a) $\vDash X \in \tau\left(\operatorname{resp} . \tau_{\alpha}, \tau_{S}, \tau_{P}, \tau_{\gamma}, \tau_{\beta}\right)$;

$\vDash \phi \in \tau\left(\right.$ resp. $\left.\tau_{\alpha}, \tau_{S}, \tau_{P}, \tau_{\gamma}, \tau_{\beta}\right)$;

b) for any $\left\{A_{\lambda}: \lambda \in \Lambda\right\} \subseteq P(X)$,

$$
\begin{aligned}
& \tau\left(\operatorname{resp} . \tau_{\alpha}, \tau_{S}, \tau_{P}, \tau_{\gamma}, \tau_{\beta}\right)\left(\bigcup_{\lambda \in \Lambda} A_{\lambda}\right) \\
& \geq \underset{\lambda \in \Lambda}{\wedge} \tau\left(\operatorname{resp} . \tau_{\alpha}, \tau_{S}, \tau_{P}, \tau_{\gamma}, \tau_{\beta}\right)\left(A_{\lambda}\right) .
\end{aligned}
$$

2) a) $\vDash X \in \tau_{C \alpha}\left(\operatorname{resp} . \tau_{C S}, \tau_{C P}, \tau_{C \gamma}, \tau_{C \beta}\right)$;

$\vDash \phi \in \tau_{C \alpha}\left(\operatorname{resp} . \tau_{C S}, \tau_{C P}, \tau_{C \gamma}, \tau_{C \beta}\right)$;

b) for any $A, B \in P(X)$,

$$
\begin{aligned}
& \tau_{C \alpha}\left(\text { resp. } \tau_{C S}, \tau_{C P}, \tau_{C \gamma}, \tau_{C \beta}\right)(A \cap B) \\
& \geq \tau_{C \alpha}\left(\operatorname{resp} . \tau_{C S}, \tau_{C P}, \tau_{C \gamma}, \tau_{C \beta}\right)(A) \\
& \wedge \tau_{C \alpha}\left(\text { resp. } \tau_{C S}, \tau_{C P}, \tau_{C \gamma}, \tau_{C \beta}\right)(B) .
\end{aligned}
$$

Theorem 3.2 1) If $\Delta$ is of type O1, then

a) $\vDash X \in \digamma_{\Delta} ; \vDash \phi \in \digamma_{\Delta}$;

b) $\vDash X \in \digamma_{C \Delta} ; \vDash \phi \in \digamma_{C \Delta}$;

2) If $\Delta$ is monotone, then

a) for any $\left\{A_{\lambda}: \lambda \in \Lambda\right\} \subseteq P(X)$,

$$
\digamma_{\Delta}\left(\bigcap_{\lambda \in \Lambda} A_{\lambda}\right) \geq \underset{\lambda \in \Lambda}{\wedge} \digamma_{\Delta}\left(A_{\lambda}\right) .
$$

b) for any $A, B \in P(X)$,

$$
\digamma_{C \Delta}(A \cap B) \geq \digamma_{C \Delta}(A) \wedge \digamma_{C \Delta}(B) .
$$

Proof. It is immediate from Theorem 3.1.

Corollary 3.2 1) a) $\vDash X \in \digamma\left(\operatorname{resp} . \digamma_{\alpha}, \digamma_{S}, \digamma_{P}, \digamma_{\gamma}, \digamma_{\beta}\right)$;

$\vDash \phi \in \digamma\left(\operatorname{resp} . \digamma_{\alpha}, \digamma_{S}, \digamma_{P}, \digamma_{\gamma}, \digamma_{\beta}\right)$;

b) $\vDash X \in \digamma_{C \alpha}\left(\operatorname{resp} . \digamma_{C S}, \digamma_{C P}, \digamma_{C \gamma}, \digamma_{C \beta}\right)$; 
$\vDash \phi \in \digamma_{C \alpha}\left(\operatorname{resp} . \digamma_{C S}, \digamma_{C P}, \digamma_{C \gamma}, \digamma_{C \beta}\right)$;

2) a) for any $\left\{A_{\lambda}: \lambda \in \Lambda\right\} \subseteq P(X)$,

$$
\begin{aligned}
& \digamma\left(\operatorname{resp} . \digamma_{\alpha}, \digamma_{S}, \digamma_{P}, \digamma_{\gamma}, \digamma_{\beta}\right)\left(\bigcap_{\lambda \in \Lambda} A_{\lambda}\right) \\
& \geq \wedge\left(\operatorname{resp} . \digamma_{\alpha}, \digamma_{S}, \digamma_{P}, \digamma_{\gamma}, \digamma_{\beta}\right)\left(A_{\lambda}\right) .
\end{aligned}
$$

b) for any $A, B \in P(X)$,

$$
\begin{aligned}
& \digamma_{C \alpha}\left(\operatorname{resp} . \digamma_{C S}, \digamma_{C P}, \digamma_{C \gamma}, \digamma_{C \beta}\right)(A \cap B) \\
& \geq \digamma_{C \alpha}\left(\operatorname{resp} . \digamma_{C S}, \digamma_{C P}, \digamma_{C \gamma}, \digamma_{C \beta}\right)(A) \\
& \wedge \digamma_{C \alpha}\left(\operatorname{resp} . \digamma_{C S}, \digamma_{C P}, \digamma_{C \gamma}, \digamma_{C \beta}\right)(A)
\end{aligned}
$$

Theorem 3.3 1) $\vDash A \in \digamma_{\Delta} \leftrightarrow \forall x(x \in \nabla(A) \rightarrow x \in A)$;

2) $\vDash A \in \digamma_{C \Delta} \leftrightarrow \forall x(x \in \bar{A} \rightarrow x \in A \cup \nabla(A))$, where $\nabla$ is the dual of $\Delta$.

\section{Proof.}

$$
\text { 1) } \begin{aligned}
& {[\forall x(x \in \nabla(A) \rightarrow x \in A)] } \\
= & {[\forall x(x \in X-A \rightarrow x \in X-\nabla(A))] } \\
= & {[\forall x(x \in X-A \rightarrow x \in \Delta(X-A))] } \\
& =\tau_{\Delta}(X-A)=\left[A \in \digamma_{\Delta}\right] . \\
\text { 2) } & {[\forall x(x \in \bar{A} \rightarrow x \in A \cup \nabla(A))] } \\
= & {[\forall x(x \in(X-(A \cup(\nabla(A))) \rightarrow x \in(X-\bar{A}))]} \\
= & {\left[\forall x\left(x \in(X-A) \cap(X-\nabla(A)) \rightarrow x \in(X-A)^{\circ}\right)\right] } \\
= & {\left[\forall x\left(x \in(X-A) \cap(\Delta(X-A)) \rightarrow x \in(X-A)^{\circ}\right)\right] } \\
= & \tau_{C \Delta}(X-A)=\left[A \in \digamma_{C \Delta}\right] .
\end{aligned}
$$

\section{Corollary 3.3}

1) $\vDash A \in \digamma\left(\operatorname{resp} . \digamma_{\alpha}, \digamma_{S}, \digamma_{P}, \digamma_{\gamma}, \digamma_{\beta}\right)$

$$
\begin{aligned}
& \leftrightarrow \forall x\left(x \in A ^ { - } \left(\text { resp. } A^{-\circ-}, A^{-\circ}, A^{\circ-},\right.\right. \\
& \left.A^{-\circ} \cup A^{\circ-}, A^{\circ-\circ} \rightarrow x \in A\right) ;
\end{aligned}
$$

2) $\vDash A \in \digamma_{C \alpha}\left(\operatorname{resp} . \digamma_{C S}, \digamma_{C P}, \digamma_{C \gamma}, \digamma_{C \beta}\right)$

$$
\begin{aligned}
& \leftrightarrow \forall x\left(x \in \bar{A} \rightarrow x \in A \cup A^{-\circ-}\right. \\
& \left.\left(\operatorname{resp} . A^{-\circ}, A^{\circ-}, A^{-\circ} \cup A^{\circ-}, A^{\circ-\circ}\right)\right) .
\end{aligned}
$$

Theorem 3.4 Let $\Delta_{1}, \Delta_{2} \in \mathfrak{I}(X)^{P(X)}$.

1) If $\Delta_{1} \leq \Delta_{2}$, a) $\vDash \tau_{\Delta_{1}} \subseteq \tau_{\Delta_{2}}$; b) $\vDash \digamma_{\Delta_{1}} \subseteq \digamma_{\Delta_{2}}$; c) $\vDash \tau_{C \Delta_{1}} \subseteq \tau_{C \Delta_{2}} ;$ d) $\vDash \digamma_{C \Delta_{1}} \subseteq \digamma_{C \Delta_{2}}$;

2) a) $\vDash \tau \subseteq \tau_{C \Delta}$; b) $\vDash \digamma \subseteq \digamma_{C \Delta}$.

Proof.

1) a) $\tau_{\Delta_{1}}(A)=\left[A \subseteq \Delta_{1}(A)\right] \leq\left[A \subseteq \Delta_{2}(A)\right]=\tau_{\Delta_{2}}(A)$; b) From a) above, we have $\digamma_{\Delta_{1}}(A)=\tau_{\Delta_{1}}(X-A) \leq \tau_{\Delta_{2}}(X-A)=\digamma_{\Delta_{2}}(A)$;

c) $\tau_{C \Delta_{1}}(A)=\left[A \cap \Delta_{1}(A) \subseteq A^{\circ}\right] \geq\left[A \cap \Delta_{2}(A) \subseteq A^{\circ}\right]$

$$
=\tau_{C \Delta_{2}}(A) \text {; }
$$

d) From (c) above, we have

$\digamma_{C \Delta_{1}}(A)=\tau_{C \Delta_{1}}(X-A) \leq \tau_{C \Delta_{2}}(X-A)=\digamma_{\Delta_{2}}(A)$.

2) a) $\tau(A)=\left[A \subseteq A^{\circ}\right] \leq\left[A \cap \Delta(A) \subseteq A^{\circ}\right]=\tau_{C \Delta}(A)$;

b) From a) above, we have

$\digamma(A)=\tau(X-A) \leq \tau_{C \Delta}(X-A)=\digamma_{C \Delta}(A)$

Corollary 3.4 1) a) i) $\vDash \tau \subseteq \tau_{\alpha}$; ii) $\vDash \tau_{\alpha} \subseteq \tau_{S}$; iii) $\vDash \tau_{\alpha} \subseteq \tau_{P}$; iv) $\vDash \tau_{S} \subseteq \tau_{\gamma}$; (v) $\vDash \tau_{P} \subseteq \tau_{\gamma}$; (vi) $\vDash \tau_{\gamma} \subseteq \tau_{\beta}$.

b) i) $\vDash \digamma \subseteq \digamma_{\alpha}$; ii) $\vDash \digamma_{\alpha} \subseteq \digamma_{S}$; iii) $\vDash \digamma_{\alpha} \subseteq \digamma_{P}$; iv) $\vDash \digamma_{S} \subseteq \digamma_{\gamma}$; v) $\vDash \digamma_{P} \subseteq \digamma_{\gamma}$; vi) $\vDash \digamma_{\gamma} \subseteq \digamma_{\beta}$.

c) i) $\vDash \tau_{C S} \subseteq \tau_{C \alpha}$; ii) $\vDash \tau_{C P} \subseteq \tau_{C \alpha}$; iii) $\vDash \tau_{C \gamma} \subseteq \tau_{C S}$; iv) $\vDash \tau_{C \gamma} \subseteq \tau_{C P}$; v) $\vDash \tau_{C \beta} \subseteq \tau_{C \gamma}$.

d) i) $\vDash \digamma_{C S} \subseteq \digamma_{C \alpha}$; ii) $\vDash \digamma_{C P} \subseteq \digamma_{C \alpha}$; iii) $\vDash \digamma_{C \gamma} \subseteq \digamma_{C S}$; iv) $\vDash \digamma_{C \gamma} \subseteq \digamma_{C P} ;$ v) $\vDash \digamma_{C \beta} \subseteq \digamma_{C \gamma}$.

2) a) $\vDash \tau \subseteq \tau_{C \alpha}\left(\operatorname{resp} . \tau_{C S}, \tau_{C P}, \tau_{C \gamma}, \tau_{C \beta}\right)$;

b) $\vDash \digamma \subseteq \digamma_{C \alpha}\left(\operatorname{resp} . \digamma_{C S}, \digamma_{C P}, \digamma_{C \gamma}, \digamma_{C \beta}\right)$.

\section{Corollary 3.5}

1) a) $\vDash \tau \subseteq \tau_{S} ; \quad$ b) $\quad \vDash \tau \subseteq \tau_{P} ; \quad$ c) $\vDash \tau \subseteq \tau_{\beta} ; \quad$ d) $\vDash \tau \subseteq \tau_{\gamma}$;

2) a) $\vDash \digamma \subseteq \digamma_{S} ;$ b) $\vDash \digamma \subseteq \digamma_{P} ;$ c) $\vDash \digamma \subseteq \digamma_{\beta}$; d) $\vDash \digamma \subseteq \digamma_{\gamma}$.

Theorem 3.5 Let $\vDash A^{\circ} \subseteq \Delta(A)$ for each $A \subseteq X$.

1) $\vDash A \in \tau_{C \Delta} \leftrightarrow A^{\circ} \equiv A \cap \Delta(A)$;

2) $\vDash A \in \digamma_{C \Delta} \leftrightarrow \bar{A} \equiv A \cup \nabla(A)$.

Proof. 1) Since $\vDash A^{\circ} \subseteq \Delta(A)$ and $\vDash A^{\circ} \subseteq A$, then $\vDash A^{\circ} \subseteq A \cap \Delta(A)$. Thus

$$
\begin{aligned}
\tau_{C \Delta}(A) & =\left[A^{\circ} \subseteq A \cap \Delta(A)\right] \wedge\left[A \cap \Delta(A) \subseteq A^{\circ}\right] \\
& =\left[A^{\circ} \equiv A \cap \Delta(A)\right] .
\end{aligned}
$$

2) Since for every $A \subseteq X, \vDash A^{\circ} \subseteq \Delta(A)$, then one can deduce that $\vDash \nabla(A) \subseteq \bar{A}$ for every $A \subseteq X$. Also, since $\vDash A \subseteq \bar{A}$ one can have that $\vDash A \cup \nabla(A) \subseteq \bar{A}$. From Theorem 3.3 (2) we have

$$
\begin{aligned}
\digamma_{C \Delta}(A) & =[\bar{A} \subseteq A \cup \nabla(A)] \\
& =[\bar{A} \subseteq A \cup \nabla(A)] \wedge[A \cup \nabla(A) \subseteq \bar{A}] \\
& =[\bar{A} \equiv A \cup \nabla(A)] .
\end{aligned}
$$

Corollary 3.6 For any $A \subseteq X$,

1) $\vDash A \in \tau_{C \alpha}\left(\right.$ resp. $\left.\tau_{C S}, \tau_{C P}, \tau_{C \gamma}, \tau_{C \beta}\right)$ $\leftrightarrow A^{\circ} \equiv A \cap A^{\circ-\circ}\left(\operatorname{resp} . A^{\circ-}, A^{-\circ}, A^{\circ-} \cup A^{-\circ}, A^{-\circ-}\right) ;$ 
2) $\vDash A \in \digamma_{C \alpha}\left(\operatorname{resp} . \digamma_{C S}, \digamma_{C P}, \digamma_{C \gamma}, \digamma_{C \beta}\right)$

$\leftrightarrow \bar{A} \equiv A \cup A^{-\circ-}\left(\operatorname{resp} . A^{-\circ}, A^{\circ-}, A^{-\circ} \cup A^{\circ-}, A^{\circ-\circ}\right)$.

Remark 3.1 In crisp setting, i.e., if the underlying fuzzifying topology is the ordinary topology, one can have

$$
\begin{aligned}
& \vDash A \in \tau \leftrightarrow\left(A \in \tau_{\alpha} \wedge A \in \tau_{C \alpha}\right) \\
& \left(\operatorname{resp} .\left(A \in \tau_{S} \wedge A \in \tau_{C S}\right),\right. \\
& \left(A \in \tau_{P} \wedge A \in \tau_{C P}\right),\left(A \in \tau_{\gamma} \wedge A \in \tau_{C \gamma}\right), \\
& \left(A \in \tau_{\beta} \wedge A \in \tau_{C \beta}\right) .(*)
\end{aligned}
$$

Of course the implication " $\rightarrow$ " in $(*)$ is either the Łukaciewicz's implication or the Boolean's implication since these implications are identical in crisp setting. But in fuzzifying setting the statement $\left(^{*}\right)$ may not be true as illustrated by the following example.

Example 3.1 Let $X=\{a, b, c\}$ and $\tau$ be a fuzzifying topology on $X$ defined as follows:

$$
\begin{gathered}
\tau(X)=\tau(\phi)=\tau(\{a\})=\tau(\{a, c\})=1 ; \\
\tau(\{b\})=\tau(\{a, b\})=0 \text { and } \tau(\{c\})=\tau(\{b, c\})=\frac{1}{4} .
\end{gathered}
$$

From the definitions of the interior and the closure of a subset of $X$ and the definitions of the interior and the closure of a fuzzy subset of $X$ and some calculations we have:

$$
\begin{aligned}
\tau_{\alpha}(\{a, b\}) & =\tau_{S}(\{a, b\})=\tau_{P}(\{a, b\}) \\
& =\tau_{\gamma}(\{a, b\})=\tau_{\beta}(\{a, b\})=\frac{3}{4}
\end{aligned}
$$

and

$$
\begin{aligned}
\tau_{C \alpha}(\{a, b\}) & =\tau_{C S}(\{a, b\})=\tau_{C P}(\{a, b\}) \\
& =\tau_{C \gamma}(\{a, b\})=\tau_{C \beta}(\{a, b\})=\frac{1}{4} .
\end{aligned}
$$

So,

$$
\begin{aligned}
& \tau_{\alpha}(\{a, b\}) \wedge \tau_{C \alpha}(\{a, b\}) \\
& =\tau_{S}(\{a, b\}) \wedge \tau_{C S}(\{a, b\}) \\
& =\tau_{P}(\{a, b\}) \wedge \tau_{C P}(\{a, b\}) \\
& =\tau_{\gamma}(\{a, b\}) \wedge \tau_{C \gamma}(\{a, b\}) \\
& =\tau_{\beta}(\{a, b\}) \wedge \tau_{C \beta}(\{a, b\}) \\
& =\frac{3}{4} \wedge \frac{1}{4}=\frac{1}{4} \neq 0=\tau(\{a, b\}) .
\end{aligned}
$$

Theorem 3.6 Let $\vDash A^{\circ} \subseteq \Delta(A)$ for each $A \subseteq X$. Then

1) $\vDash A \in \tau \rightarrow\left(A \in \tau_{\Delta} \wedge A \in \tau_{C \Delta}\right)$;

2) $\vDash\left(A \in \tau_{\Delta} \otimes A \in \tau_{C \Delta}\right) \rightarrow A \in \tau$.
Proof. 1) It is obtained from Theorem 2.4 (1)(a) and (2)(a).

2) $\left[A \in \tau_{\Delta} \otimes A \in \tau_{C \Delta}\right]=\max \left(0, \tau_{\Delta}(A)+\tau_{C \Delta}(A)-1\right)$

$$
\begin{aligned}
= & \max \left(0, \inf _{x \in A} \Delta(A)(x)\right. \\
& \left.+\inf _{x \in A} \min \left(1,1-\Delta(A)(x)+A^{\circ}(x)\right)-1\right) \\
= & \max \left(0, \inf _{x \in A} \Delta(A)(x)\right. \\
& \left.+\inf _{x \in A}\left(1-\Delta(A)(x)+A^{\circ}(x)\right)-1\right) \\
\leq & \max \left(0, \inf _{x \in A}\left(\Delta(A)(x)+1-\Delta(A)(x)+A^{\circ}(x)-1\right)\right) \\
= & \inf _{x \in A} A^{\circ}(x)=[A \in \tau] .
\end{aligned}
$$

\section{Corollary 3.7}

1) $\vDash A \in \tau$

$\rightarrow\left(A \in \tau_{\alpha} \wedge A \in \tau_{C \alpha}\right)\left(\operatorname{resp} .\left(A \in \tau_{S} \wedge A \in \tau_{C S}\right)\right.$,

$\left(A \in \tau_{P} \wedge A \in \tau_{C P}\right),\left(A \in \tau_{\gamma} \wedge A \in \tau_{C \gamma}\right)$;

$\left.\left(A \in \tau_{\beta} \wedge A \in \tau_{C \beta}\right)\right)$;

2) $\vDash\left(A \in \tau_{\alpha} \otimes A \in \tau_{C \alpha}\right)\left(\operatorname{resp} .\left(A \in \tau_{S} \otimes A \in \tau_{C S}\right)\right.$,

$$
\begin{aligned}
& \left(A \in \tau_{P} \otimes A \in \tau_{C P}\right),\left(A \in \tau_{\gamma} \otimes A \in \tau_{C \gamma}\right) ; \\
& \left.\left(A \in \tau_{\beta} \otimes A \in \tau_{C \beta}\right)\right) \rightarrow A \in \tau .
\end{aligned}
$$

Theorem 3.7 Let $\vDash A^{\circ} \subseteq \Delta(A)$ for each $A \subseteq X$. If for every $A \subseteq X, \vDash A \in \tau_{\Delta}$ or $\vDash A \in \tau_{C \Delta}$, then

1) $\vDash A \in \tau \leftrightarrow\left(A \in \tau_{\Delta} \wedge A \in \tau_{C \Delta}\right)$;

2) $\vDash\left(A \in \tau_{\Delta} \otimes A \in \tau_{C \Delta}\right) \leftrightarrow A \in \tau$.

Proof. Using Theorem 3.6 it remains to prove the following

1) Suppose that $\vDash A \in \tau_{\Delta}$. Then for each $x \in A$ we have $\Delta(A)(x)=1$. So

$$
\begin{aligned}
& {\left[A \in \tau_{\Delta}\right] \wedge\left[A \in \tau_{C \Delta}\right]=\left[A \in \tau_{C \Delta}\right]} \\
& =\inf _{x \in A} \min \left(1,1-\Delta(A)(x)+A^{\circ}(x)\right) \\
& =\inf _{x \in A} A^{\circ}(x)=\tau(A) .
\end{aligned}
$$

Now, suppose that $\vDash A \in \tau_{C \Delta}$. Since $\left[A^{\circ} \subseteq \Delta(A)\right]=1$, then

$$
\begin{aligned}
1 & =\min \left(1,1-\Delta(A)(x)+A^{\circ}(x)\right) \\
& =1-\Delta(A)(x)+A^{\circ}(x) .
\end{aligned}
$$

For each $x \in A$ we have $\Delta(A)(x)=A^{\circ}(x)$. Thus,

$$
\left[A \in \tau_{\Delta}\right] \wedge\left[A \in \tau_{C \Delta}\right]=\left[A \in \tau_{\Delta}\right]=\tau(A) .
$$

2) The proof is similar to 1 ).

Corollary 3.8 If for every $A \subseteq X, \vDash A \in \tau_{\alpha}$ or $\vDash A \in \tau_{C \alpha} \quad$ (resp. $\vDash A \in \tau_{S}$ or $\vDash A \in \tau_{C S}, \quad \vDash A \in \tau_{P}$ or $\vDash A \in \tau_{C P}, \vDash A \in \tau_{\gamma}$ or $\vDash A \in \tau_{C \gamma}, \vDash A \in \tau_{\beta}$ or 
$\left.\vDash A \in \tau_{C \beta}\right)$, then

1) $\vDash A \in \tau$

$$
\begin{aligned}
& \leftrightarrow\left(A \in \tau_{\alpha} \wedge A \in \tau_{C \alpha}\right)\left(\operatorname{resp} .\left(A \in \tau_{S} \wedge A \in \tau_{C S}\right),\right. \\
& \left(A \in \tau_{P} \wedge A \in \tau_{C P}\right),\left(A \in \tau_{\gamma} \wedge A \in \tau_{C \gamma}\right) ; \\
& \left.\left(A \in \tau_{\beta} \wedge A \in \tau_{C \beta}\right)\right) ; \\
& \vDash A \in \tau \\
& \leftrightarrow\left(A \in \tau_{\alpha} \otimes A \in \tau_{C \alpha}\right)\left(\operatorname{resp} .\left(A \in \tau_{S} \otimes A \in \tau_{C S}\right),\right. \\
& \left(A \in \tau_{P} \otimes A \in \tau_{C P}\right),\left(A \in \tau_{\gamma} \otimes A \in \tau_{C \gamma}\right) ; \\
& \left.\left(A \in \tau_{\beta} \otimes A \in \tau_{C \beta}\right)\right) .
\end{aligned}
$$

2) $\vDash A \in \tau$

Theorem 3.8 Let $\Delta$ be a monotone. Then

$$
\vDash A \in \tau_{\Delta} \leftrightarrow \forall x\left(x \in A \rightarrow \exists B\left(B \in \tau_{\Delta} \wedge x \in B \subseteq A\right)\right) .
$$

Proof.

$$
\begin{aligned}
& {\left[\forall x\left(x \in A \rightarrow \exists B\left(B \in \tau_{\Delta} \wedge x \in B \subseteq A\right)\right)\right]} \\
& =\inf _{x \in A} \sup _{x \in B \subseteq A} \tau_{\Delta}(B) .
\end{aligned}
$$

First, we have $\inf _{x \in A} \sup _{x \in B \subseteq A} \tau_{\Delta}(B) \geq \tau_{\Delta}(A)$. On the other hand, Suppose that $\beta_{x}=\{B: x \in B \subseteq A\}$. Then for any $f \in \prod_{x \in A} \beta_{x}$, we have $\bigcup_{x \in A} f(x)=A$. Since $\Delta$ is monotone, then by Theorem 3.1 (2)(a) we have $\tau_{\Delta}(A)=\tau_{\Delta}\left(\bigcup_{x \in A} f(x)\right) \geq \inf _{x \in A} \tau_{\Delta}(f(x))$. By completely distributive law we have

$$
\tau_{\Delta}(A) \geq \sup _{f \in \prod_{x \in A} \beta_{x}} \inf _{x \in A} \tau_{\Delta}(f(x))=\inf _{x \in A} \sup _{x \in B \subseteq A} \tau_{\Delta}(B) .
$$

\section{Corollary 3.9}

$$
\begin{aligned}
& \vDash A \in \tau\left(\operatorname{resp} . \tau_{\alpha}, \tau_{S}, \tau_{P}, \tau_{\gamma}, \tau_{\beta}\right) \leftrightarrow \forall x(x \in A \\
& \rightarrow \exists B\left(B \in \tau\left(\operatorname{resp} . \tau_{\alpha}, \tau_{S}, \tau_{P}, \tau_{\gamma}, \tau_{\beta}\right) \wedge x \in B \subseteq A\right) .
\end{aligned}
$$

Remark 3.2 The following are valid in crisp setting:

1) $\vDash A \in \tau_{\alpha} \wedge B \in \tau_{\alpha} \rightarrow A \cap B \in \tau_{\alpha}$;

2) $\vDash A \in \tau \wedge B \in \tau_{\gamma} \rightarrow A \cap B \in \tau_{\gamma}$;

3) $\vDash A \in \tau_{\alpha} \wedge B \in \tau_{\gamma} \rightarrow A \cap B \in \tau_{\gamma}$,

but in fuzzifying setting these statement may not be true by the following example.

Example 3.2 From Example 3.1 we have

$$
\text { 1) } \begin{aligned}
& \tau_{\alpha}(\{a, b\}) \wedge \tau_{\alpha}(\{b, c\})=\frac{1}{4} \not \leq 0 \\
= & \tau_{\alpha}(\{b\})=\tau_{\alpha}(\{a, b\} \cap\{b, c\}) . \\
\text { 2) } & \tau(\{b, c\}) \wedge \tau_{\gamma}(\{a, b\})=\frac{1}{4} \not \leq 0 \\
= & \tau_{\gamma}(\{b\})=\tau_{\gamma}(\{a, b\} \cap\{b, c\}) .
\end{aligned}
$$

$$
\text { 3) } \begin{aligned}
& \tau_{\alpha}(\{a, b\}) \wedge \tau_{\gamma}(\{b, c\})=\frac{1}{4} \not \leq 0 \\
= & \tau_{\gamma}(\{b\})=\tau_{\gamma}(\{a, b\} \cap\{b, c\}) .
\end{aligned}
$$

\section{Fuzzifying Neighborhood Structure of a Point}

In this section the concepts of $\Delta$-neighborhood system and $C \Delta$-neighborhood system of a point are presented and a fuzzifying topology induced by $C \Delta$-neighborhood system is obtained.

Definition 4.1 The fuzzifying $\Delta$-(resp. CA-) neighborhood system of $x \in X$, denoted by $N_{x}^{\Delta}$ (resp. $N_{x}^{C \Delta}$ ) $\in \mathfrak{I}(P(X))$, is defined as follows:

$$
\begin{aligned}
& A \in N_{x}^{\Delta}:=\exists B\left(B \in \tau_{\Delta} \wedge x \in B \subseteq A\right) \\
& \quad\left(\text { resp. } A \in N_{x}^{C \Delta}:=\exists B\left(B \in \tau_{C \Delta} \wedge x \in B \subseteq A\right)\right), \\
& N_{x}^{\Delta}(A)=\sup _{x \in B \subseteq A} \tau_{\Delta}(B)\left(\operatorname{resp.} N_{x}^{C \Delta}(A)=\sup _{x \in B \subseteq A} \tau_{C \Delta}(B)\right) .
\end{aligned}
$$$$
\text { i.e., }
$$

Definition 4.2 Let $x \in X$.

1) If $\Delta=()^{\circ},\left(\operatorname{resp} .()^{\circ-\circ},()^{\circ-},()^{-\circ},()^{-\circ} \cup()^{\circ-},()^{-\circ-}\right)$, the fuzzifying $\Delta$-neighborhood system of $x$ coincides with the fuzzifying (resp. fuzzifying $\alpha$-, fuzzifying semi-, fuzzifying pre-, fuzzifying $\gamma-$, fuzzifying $\beta$-) neighborhood system of $x$ and will be denoted by $N_{x}$ (resp. $\left.N_{x}^{\alpha}, N_{x}^{S}, N_{x}^{P}, N_{x}^{\gamma}, N_{x}^{\beta}\right)$;

2) If $\Delta=0^{\circ-\circ}\left(\right.$ resp. ()$\left.^{\circ-},()^{-\circ},()^{-\circ} \cup()^{\circ-},()^{-\circ-}\right)$, the fuzzifying $C \Delta$-neighborhood system of $x$ coincides with the fuzzifying $c \alpha$ - (resp. fuzzifying csemi-, fuzzifying cpre-, fuzzifying $c \gamma$-, fuzzifying $c \beta$-) neighborhood system of $x$ and will be denoted by $N_{x}^{C \alpha}$ (resp. $\left.N_{x}^{C S}, N_{x}^{C P}, N_{x}^{C \gamma}, N_{x}^{C \beta}\right)$.

Theorem 4.1 Let $\Delta$ be a monotone.
1) $\vDash A \in \tau_{\Delta} \leftrightarrow \forall x\left(x \in A \rightarrow \exists B\left(B \in N_{x}^{\Delta} \wedge B \subseteq A\right)\right)$;
2) $\vDash \forall x\left(x \in A \rightarrow A \in N_{x}^{\Delta}\right) \leftrightarrow A \in \tau_{\Delta}$.

Proof. Using Theorem 3.8 we have

$$
\begin{aligned}
& {\left[\forall x\left(x \in A \rightarrow \exists B\left(B \in N_{x}^{\Delta} \wedge B \subseteq A\right)\right)\right]} \\
& =\inf _{x \in A} \sup _{B \subseteq A} N_{x}^{\Delta}(B)=\inf _{x \in A} \sup _{B \subseteq A} \sup _{x \in C \subseteq B} \tau_{\Delta}(C) \\
& =\inf _{x \in A} \sup _{x \in C \subseteq A} \tau_{\Delta}(C)=\left[A \in \tau_{\Delta}\right] .
\end{aligned}
$$

2) From 1) the proof is immediate.

Corollary 4.1 Let $(X, \tau)$ be a fuzzifying topological space.

$$
\text { 1) } \begin{aligned}
\tau & (A)\left(\operatorname{resp} . \tau_{\alpha}(A), \tau_{S}(A), \tau_{P}(A), \tau_{\gamma}(A), \tau_{\beta}(A)\right) \\
= & \inf _{x \in A} \sup _{x \in C \subseteq A} \tau(C)\left(\operatorname{resp} . \tau_{\alpha}(C), \tau_{S}(C),\right. \\
& \left.\tau_{P}(C), \tau_{\gamma}(C), \tau_{\beta}(C)\right) ;
\end{aligned}
$$


1) $\tau(A)\left(\operatorname{resp} . \tau_{\alpha}(A), \tau_{S}(A), \tau_{P}(A), \tau_{\gamma}(A), \tau_{\beta}(A)\right)$

$$
\begin{aligned}
= & \inf _{x \in A} N_{x}(A)\left(\operatorname{resp} . N_{x}^{\alpha}(A), N_{x}^{S}(A),\right. \\
& \left.N_{x}^{P}(A), N_{x}^{\gamma}(A), N_{x}^{\beta}(A)\right) ;
\end{aligned}
$$

Theorem 4.2 The mapping $N^{\Delta}: X \rightarrow \mathfrak{I}(P(X))$, $x \mapsto N_{x}^{\Delta}$, has the following properties:

1) If $\Delta$ is of type $O 1$, then $N_{x}^{\Delta}$ is normal for any $x \in X$;

2) For any $x, A, \vDash A \in N_{x}^{\Delta} \rightarrow x \in A$;

3) For any $x, A, B, \vDash A \subseteq B \rightarrow\left(A \in N_{x}^{\Delta} \rightarrow B \in N_{x}^{\Delta}\right)$;

4) If $\Delta$ is monotone, then for any $x, A$,

$\vDash A \in N_{x}^{\Delta}$

$\rightarrow \exists H\left(H \in N_{x}^{\Delta} \wedge H \subseteq A \wedge \forall y\left(y \in H \rightarrow H \in N_{y}^{\Delta}\right)\right) ;$

5) If $\Delta$ is of type $O 2$, then

$$
\vDash A \in N_{x}^{\Delta} \wedge B \in N_{x}^{\Delta} \rightarrow A \cap B \in N_{x}^{\Delta} .
$$

Proof. 1) Since $\Delta$ is of type $O 1$, then

$$
N_{x}^{\Delta}(X)=\sup _{x \in B \subseteq X} \tau_{\Delta}(B) \geq \tau_{\Delta}(X)=[X \subseteq \Delta(X)]=1 .
$$

2) If $\left[A \in N_{x}^{\Delta}\right]=0$, then the results holds. Suppose $\left[A \in N_{x}^{\Delta}\right]=\sup _{x \in H \subseteq A} \tau_{\Delta}(H)>0$. Then there exists

$H_{\mathrm{o}} \in P(X)$ such that $x \in H_{\mathrm{o}} \subseteq A$. Now, we have $[x \in A]=1$. Thus $\left[A \in N_{x}^{\Delta}\right] \leq[x \in A]$ holds always.

3) If $[A \subseteq B]=0$, then the result holds. Now, suppose that $[A \subseteq B]=1$. Then we have

$$
\left[A \in N_{x}^{\Delta}\right]=\sup _{x \in C \subseteq A} \tau_{\Delta}(C) \leq \sup _{x \in C \subseteq B} \tau_{\Delta}(C)=\left[B \in N_{x}^{\Delta}\right] .
$$

4) Since $\Delta$ is monotone, then from Theorem 4.1 a) we have

$$
\begin{aligned}
& {\left[\exists H\left(H \in N_{x}^{\Delta} \wedge H \subseteq A \wedge \forall y\left(y \in H \rightarrow H \in N_{y}^{\Delta}\right)\right)\right]} \\
& =\sup _{H \subseteq A}\left(N_{x}^{\Delta}(H) \wedge \inf _{y \in H} N_{y}^{\Delta}(H)\right)=\sup _{H \subseteq A}\left(N_{x}^{\Delta}(H) \wedge \tau_{\Delta}(H)\right) \\
& =\sup _{H \subseteq A} \tau_{\Delta}(H) \geq \sup _{x \in H \subseteq A} \tau_{\Delta}(H)=\left[A \in N_{x}^{\Delta}\right] . \\
& \text { 5) } N_{x}^{\Delta}(A \cap B)=\sup _{x \in C \subseteq A \cap B} \tau_{\Delta}(C) \\
& =\sup _{x \in C_{1} \subseteq A, x \in C_{2} \subseteq B} \tau_{\Delta}\left(C_{1} \cap C_{2}\right) \\
& \geq \sup _{x \in C_{1} \subseteq A, x \in C_{2} \subseteq B}\left(\tau_{\Delta}\left(C_{1}\right) \wedge \tau_{\Delta}\left(C_{2}\right)\right) \\
& =\sup _{x \in C_{1} \subseteq A} \tau_{\Delta}\left(C_{1}\right) \wedge \sup _{x \in C_{2} \subseteq B} \tau_{\Delta}\left(C_{2}\right) \\
& =N_{x}^{\Delta}(A) \wedge N_{x}^{\Delta}(B) .
\end{aligned}
$$

\section{Corollary 4.2 The mapping $N$}

$$
\begin{gathered}
\left(\operatorname{resp} . N^{\alpha}, N^{S}, N^{P}, N^{\gamma}, N^{\beta}\right): X \rightarrow \Im(P(X)), \\
x \mapsto N_{x}\left(\text { resp. } N_{x}^{\alpha}, N_{x}^{S}, N_{x}^{P}, N_{x}^{\gamma}, N_{x}^{\beta}\right),
\end{gathered}
$$

has the following properties:

1) For any $x \in X, N_{x}\left(\operatorname{resp} . N_{x}^{\alpha}, N_{x}^{S}, N_{x}^{P}, N_{x}^{\gamma}, N_{x}^{\beta}\right)$ is normal;

2) For any $x, A$,

$$
\vDash A \in N_{x}\left(\operatorname{resp} . N_{x}^{\alpha}, N_{x}^{S}, N_{x}^{P}, N_{x}^{\gamma}, N_{x}^{\beta}\right) \rightarrow x \in A ;
$$

3) For any $x, A, B$,

$$
\begin{aligned}
& \vDash A \subseteq B \rightarrow\left(A \in N_{x}\left(\operatorname{resp} . N_{x}^{\alpha}, N_{x}^{S}, N_{x}^{P}, N_{x}^{\gamma}, N_{x}^{\beta}\right)\right. \\
& \left.\rightarrow B \in N_{x}\left(\operatorname{resp} . N_{x}^{\alpha}, N_{x}^{S}, N_{x}^{P}, N_{x}^{\gamma}, N_{x}^{\beta}\right)\right) ;
\end{aligned}
$$

4) For any $x, A$,

$$
\vDash A \in N_{x}\left(\operatorname{resp} . N_{x}^{\alpha}, N_{x}^{S}, N_{x}^{P}, N_{x}^{\gamma}, N_{x}^{\beta}\right)
$$$$
\rightarrow \exists H\left(H \in N_{x}\left(\operatorname{resp} . N_{x}^{\alpha}, N_{x}^{S}, N_{x}^{P}, N_{x}^{\gamma}, N_{x}^{\beta}\right) \wedge H\right.
$$$$
\left.\subseteq A \wedge \forall y\left(y \in H \rightarrow H \in N_{x}\left(\operatorname{resp} . N_{x}^{\alpha}, N_{x}^{S}, N_{x}^{P}, N_{x}^{\gamma}, N_{x}^{\beta}\right)\right)\right) \text {; }
$$

5) For any $x, A, B$,

$$
\vDash A \in N_{x} \wedge B \in N_{x} \rightarrow A \cap B \in N_{x} .
$$

Theorem 4.3 The mapping $N^{C \Delta}: X \rightarrow \mathfrak{J}(P(X))$, $x \mapsto N_{x}^{C \Delta}$, has the following properties:

1) If $\Delta$ is of type $O 1$, then $N_{x}^{C \Delta}$ is normal for any $x \in X$;

2) For any $x, A, \vDash A \in N_{x}^{C \Delta} \rightarrow x \in A$;

3) For any $x, A, B$,

$$
\vDash A \subseteq B \rightarrow\left(A \in N_{x}^{C \Delta} \rightarrow B \in N_{x}^{C \Delta}\right) ;
$$

4) If $\Delta$ is monotone, then or any $x, A, B$,

$$
\vDash A \in N_{x}^{C \Delta} \wedge B \in N_{x}^{C \Delta} \rightarrow A \cap B \in N_{x}^{C \Delta} .
$$

Conversely, if a mapping $N^{C \Delta}$ satisfies 1), 3) and 4), then it assigns a fuzzifying topology on $X$, denoted by $\tau_{N^{c \Delta}} \in \mathfrak{I}(P(X))$, is defined as follows:

$$
A \in \tau_{N^{C \Delta}}:=\forall x\left(x \in A \rightarrow A \in N_{x}^{C \Delta}\right) .
$$

Proof. Since $N_{x}^{C \Delta}$ is normal and satisfies properties (2) and (3) in Theorem 4.2, then $\tau_{N^{C \Delta}}$ is a fuzzifying topology on $X$. The rest of the proof is similar to the proof of Theorem 4.2.

Corollary 4.3 The mapping $N^{C \alpha}$

$$
\begin{gathered}
\left(\operatorname{resp} . N^{C S}, N^{C P}, N^{C \gamma}, N^{C \beta}\right): X \rightarrow \Im(P(X)), x \mapsto N_{x}^{C \alpha} \\
\left(\operatorname{resp} . N_{x}^{C S}, N_{x}^{C P}, N_{x}^{C \gamma}, N_{x}^{C \beta}\right),
\end{gathered}
$$

has the following properties:

1) $N_{x}^{C \alpha}\left(\operatorname{resp} . N_{x}^{C S}, N_{x}^{C P}, N_{x}^{C \gamma}, N_{x}^{C \beta}\right)$ is normal for any $x \in X$

2) For any $x, A$, 


$$
\vDash A \in N_{x}^{C \alpha}\left(\operatorname{resp} . N_{x}^{C S}, N_{x}^{C P}, N_{x}^{C \gamma}, N_{x}^{C \beta}\right) \rightarrow x \in A ;
$$

3) For any $x, A, B$,

$$
\begin{aligned}
& \vDash A \subseteq B \rightarrow\left(A \in N_{x}^{C \alpha}\left(\operatorname{resp} . N_{x}^{C S}, N_{x}^{C P}, N_{x}^{C \gamma}, N_{x}^{C \beta}\right)\right. \\
& \left.\rightarrow B \in N_{x}^{C \alpha}\left(\operatorname{resp} . N_{x}^{C S}, N_{x}^{C P}, N_{x}^{C \gamma}, N_{x}^{C \beta}\right)\right) ;
\end{aligned}
$$

4) If $\Delta$ is monotone, then or any $x, A, B$,

$$
\begin{aligned}
& \vDash A \in N_{x}^{C \alpha}\left(\operatorname{resp} . N_{x}^{C S}, N_{x}^{C P}, N_{x}^{C \gamma}, N_{x}^{C \beta}\right) \\
& \wedge B \in N_{x}^{C \alpha}\left(\operatorname{resp} . N_{x}^{C S}, N_{x}^{C P}, N_{x}^{C \gamma}, N_{x}^{C \beta}\right) \\
& \rightarrow A \cap B \in N_{x}^{C \alpha}\left(\operatorname{resp} . N_{x}^{C S}, N_{x}^{C P}, N_{x}^{C \gamma}, N_{x}^{C \beta}\right) .
\end{aligned}
$$

5) The mapping $N^{C \alpha}\left(\operatorname{resp} . N^{C S}, N^{C P}, N^{C \gamma}, N^{C \beta}\right)$ assigns a fuzzifying topology on $\mathrm{X}$, denoted by $\tau_{N^{C \alpha}}$ (resp. $\left.\tau_{N^{C S}}, \tau_{N}{ }_{N P}, \tau_{N^{C \gamma}}, \tau_{N^{C \beta}}\right) \in \mathfrak{I}(P(X))$, is defined as follows:

$$
\begin{aligned}
& A \in \tau_{N^{C \alpha}}\left(\operatorname{resp} . \tau_{N^{C S}}, \tau_{N^{C P}}, \tau_{N^{C \gamma}}, \tau_{N^{C \beta}}\right) \\
& :=\forall x\left(x \in A \rightarrow A \in N_{x}^{C \alpha}\left(\operatorname{resp} . N_{x}^{C S}, N_{x}^{C P}, N_{x}^{C \gamma}, N_{x}^{C \beta}\right)\right) .
\end{aligned}
$$

Theorem 4.4 If $\Delta$ is of type $O 1$ and monotone, then $\vDash \tau_{C \Delta} \subseteq \tau_{N^{C \Delta}}$.

Proof. Let $B \subseteq X$. Then

$$
\tau_{N^{C \Delta}}(B)=\inf _{x \in B} N_{x}^{C \Delta}(B)=\inf _{x \in B} \sup _{x \in A \subseteq B} \tau_{C \Delta}(A) \geq \tau_{C \Delta}(B) .
$$

Corollary 4.4 1) $\vDash \tau_{C \alpha} \subseteq \tau_{N^{C \alpha}}$; 2) $\vDash \tau_{C S} \subseteq \tau_{N^{C S}}$; 3)

$$
\left.\vDash \tau_{C P} \subseteq \tau_{N}{ }_{N} ; 4\right) \vDash \tau_{C \gamma} \subseteq \tau_{N}{ }_{N \gamma} ;(5) \vDash \tau_{C \beta} \subseteq \tau_{N^{C \beta}} \text {. }
$$

\section{Closure and Interior Operations in Fuzzifying Topology}

The purpose of this section is to establish the concepts of fuzzifying $\Delta$ - and $C \Delta$-derived sets, fuzzifying $\Delta$ - and $C \Delta$-closure operation and fuzzifying $\Delta$ - and $C \Delta$-interior operation and study some of their properties.

Definition 5.1 The fuzzifying $\Delta$ - (resp. C $\Delta$-) derived set $d_{\Delta}(A)$ (resp. $\left.d_{C \Delta}(A)\right) \in \mathfrak{J}(X)$ of $A \subseteq X$ is defined as follows:

$$
\begin{aligned}
& \qquad x \in d_{\Delta}(A):=\forall B\left(B \in N_{x}^{\Delta} \rightarrow B \cap(A-\{x\}) \neq \phi\right) \\
& \text { i.e., } \\
& \text { resp. } \left.x \in d_{C \Delta}(A):=\forall B\left(B \in N_{x}^{C \Delta} \rightarrow B \cap(A-\{x\}) \neq \phi\right)\right), \\
& \quad d_{\Delta}(A)(x)=\inf _{B \cap(A-\{x\})=\phi}\left(1-N_{x}^{\Delta}(B)\right) \\
& \left(\operatorname{resp} . d_{C \Delta}(A)(x)=\inf _{B \cap(A-\{x\})=\phi}\left(1-N_{x}^{C \Delta}(B)\right)\right) .
\end{aligned}
$$

Definition 5.2 For $A \subseteq X$.
1) If $\Delta=()^{\circ},\left(\operatorname{resp} .()^{\circ-\circ},()^{\circ-},()^{-\circ},()^{-\circ} \cup()^{\circ-},()^{-\circ-}\right)$, the notion of fuzzifying $\Delta$-derived set of $A \subseteq X$ coincides with the notion of fuzzifying derived (resp. $\alpha$ derived, semi-derived, pre-derived, $\gamma$-derived, $\beta$-derived) set and will be denoted by

$$
A^{\prime}\left(\operatorname{resp} . d_{\alpha}(A), d_{S}(A), d_{P}(A), d_{\gamma}(A), d_{\beta}(A)\right) ;
$$

2) If $\Delta=0^{\circ-\circ}$ (resp. ()$\left.^{\circ-},()^{-\circ},()^{-\circ} \cup()^{\circ-},()^{-\circ-}\right)$, the notion of fuzzifying $c \Delta$-derived set of $A \subseteq X$ coincides with the notion of fuzzifying derived (resp. $c \alpha$-derived, csemi-derived, cpre-derived, $c \gamma$-derived, $c \beta$-derived) set and will be denoted by

$$
d_{C \alpha}(A)\left(\operatorname{resp} . d_{C S}(A), d_{C P}(A), d_{C \gamma}(A), d_{C \beta}(A)\right) .
$$

Theorem 5.1 For every $A \subseteq X$ we have

1) $d_{\Delta}(A)(x)=1-N_{x}^{\Delta}((X-A) \cup\{x\})$;

2) $d_{C \Delta}(A)(x)=1-N_{x}^{C \Delta}((X-A) \cup\{x\})$.

Proof. 1) Using Theorem 4.3 3) we have

$$
\begin{aligned}
d_{\Delta}(A)(x) & =\inf _{B \cap(A-\{x\})=\phi}\left(1-N_{x}^{\Delta}(B)\right) \\
& =1-\sup _{B \cap(A-\{x\})=\phi} N_{x}^{\Delta}(B) \\
& =1-\sup _{B \subseteq(X-A) \cup\{x\}} N_{x}^{\Delta}(B) \\
& =1-N_{x}^{\Delta}((X-A) \cup\{x\}) .
\end{aligned}
$$

2) It is similar to the proof. of 1).

\section{Corollary 5.1}

1) a) $A^{\prime}(x)=1-N_{x}((X-A) \cup\{x\})$;

b) $d_{\alpha}(A)(x)=1-N_{x}^{\alpha}((X-A) \cup\{x\})$;

c) $d_{S}(A)(x)=1-N_{x}^{S}((X-A) \cup\{x\})$;

d) $d_{P}(A)(x)=1-N_{x}^{P}((X-A) \cup\{x\})$;

e) $d_{\gamma}(A)(x)=1-N_{x}^{\gamma}((X-A) \cup\{x\})$;

f) $d_{\beta}(A)(x)=1-N_{x}^{\beta}((X-A) \cup\{x\})$;

2) a) $d_{C \alpha}(A)(x)=1-N_{x}^{C \alpha}((X-A) \cup\{x\})$;

b) $d_{C S}(A)(x)=1-N_{x}^{C S}((X-A) \cup\{x\})$;

c) $d_{C P}(A)(x)=1-N_{x}^{C P}((X-A) \cup\{x\})$;

d) $d_{C \gamma}(A)(x)=1-N_{x}^{C \gamma}((X-A) \cup\{x\})$;

e) $d_{C \beta}(A)(x)=1-N_{x}^{C \beta}((X-A) \cup\{x\})$;

Theorem 5.2 For every $A \subseteq X$ we have

1) If $\Delta$ is monotone, then $\vDash A \in \digamma_{\Delta} \leftrightarrow d_{\Delta}(A) \subseteq A$;

2) If $\Delta$ is of type $O 1$ and monotone, then

$$
\vDash A \in \digamma_{N}{ }_{N \Delta} \leftrightarrow d_{C \Delta}(A) \subseteq A .
$$

Proof. Using Theorem 4.1 (2) we have 
1) $\left[d_{\Delta}(A) \subseteq A\right]=\inf _{x \in X-A}\left(1-d_{\Delta}(A)(x)\right)$

$$
\begin{aligned}
& =\inf _{x \in X-A} N_{x}^{\Delta}((X-A) \cup\{x\}) \\
& =\inf _{x \in X-A} N_{x}^{\Delta}(X-A)=\tau_{\Delta}(X-A)=\digamma_{\Delta}(A) .
\end{aligned}
$$

2) It is similar to 1).

\section{Corollary $\mathbf{5 . 2}$}

1) a) $\vDash A \in \digamma \leftrightarrow A^{\prime} \subseteq A$;

b) $\vDash A \in \digamma_{\alpha} \leftrightarrow d_{\alpha}(A) \subseteq A$;

c) $\vDash A \in \digamma_{S} \leftrightarrow d_{S}(A) \subseteq A$;

d) $\vDash A \in \digamma_{P} \leftrightarrow d_{P}(A) \subseteq A$;

e) $\vDash A \in \digamma_{\gamma} \leftrightarrow d_{\gamma}(A) \subseteq A$;

f) $\vDash A \in \digamma_{\beta} \leftrightarrow d_{\beta}(A) \subseteq A$;

2) a) $\vDash A \in \digamma_{N^{C \alpha}} \leftrightarrow d_{C \alpha}(A) \subseteq A$;

b) $\vDash A \in \digamma_{N}{ }_{N S} \leftrightarrow d_{C S}(A) \subseteq A$;

c) $\vDash A \in \digamma_{N}{ }_{N P} \leftrightarrow d_{C P}(A) \subseteq A$;

d) $\vDash A \in \digamma_{N}{ }_{N \gamma} \leftrightarrow d_{C \gamma}(A) \subseteq A$;

e) $\vDash A \in \digamma_{N}{ }_{N \beta} \leftrightarrow d_{C \beta}(A) \subseteq A$;

Definition 5.3 The fuzzifying $\Delta$-(resp. $C \Delta$-) closure $C l_{\Delta}(A) \quad\left(\right.$ resp. $\left.C l_{C \Delta}(A)\right) \in \mathfrak{I}(X)$ of $A \subseteq X$ is defined as follows:

$$
x \in C l_{\Delta}(A):=\forall B\left((B \supseteq A) \wedge\left(B \in \digamma_{\Delta}\right) \rightarrow x \in B\right)
$$

(resp. $\left.x \in C l_{C \Delta}(A):=\forall B\left((B \supseteq A) \wedge\left(B \in \digamma_{C \Delta}\right) \rightarrow x \in B\right)\right)$, i.e.,

$$
\begin{gathered}
C l_{\Delta}(A)(x)=\inf _{x \notin B \supseteq A}\left(1-\digamma_{\Delta}(B)\right) \\
\left(\text { resp. } C l_{C \Delta}(A)(x)=\inf _{x \notin B \supseteq A}\left(1-\digamma_{C \Delta}(B)\right)\right) .
\end{gathered}
$$

Definition 5.4 1) If $\Delta=0^{\circ}$, (resp. ()$^{\circ-\circ},()^{\circ-},()^{-\circ}$, ()$\left.^{-\circ} \cup()^{\circ-},()^{-\circ-}\right)$, the notion of fuzzifying $\Delta$-closure of $A \subseteq X$ coincides with the notion of fuzzifying closure (resp. $\alpha$-closure, semi-closure, pre-closure, $\gamma$-closure, $\beta$-closure) operation and will be denoted by

$$
\bar{A}\left(\text { resp. } C l_{\alpha}(A), C l_{S}(A), C l_{P}(A), C l_{\gamma}(A), C l_{\beta}(A)\right) \text {; }
$$

2) If $\Delta=()^{\circ-\circ}$ (resp. ()$\left.^{\circ-},()^{-\circ},()^{-\circ} \cup()^{\circ-},()^{-\circ-}\right)$, the notion of fuzzifying $C \Delta$-closure of $A \subseteq X$ coincides with the notion of fuzzifying $c \alpha$ (resp. csemi, cpre, $c \gamma, c \beta$ )-closure operation and will be denoted by

$$
C l_{C \alpha}(A)\left(\operatorname{resp} . C l_{C S}(A), C l_{C P}(A), C l_{C \gamma}(A), C l_{C \beta}(A)\right) \text {. }
$$

Theorem 5.3 For every $A \subseteq X$ we have

1) $C l_{\Delta}(A)(x)=1-N_{x}^{\Delta}(X-A)$;

2) $C l_{C \Delta}(A)(x)=1-N_{x}^{C \Delta}(X-A)$;

\section{Proof.}

$$
\text { 1) } \begin{aligned}
C l_{\Delta}(A)(x) & =\inf _{x \notin B \supseteq A}\left(1-\digamma_{\Delta}(B)\right) \\
& =\inf _{x \in X-B \subseteq X-A}\left(1-\tau_{\Delta}(X-B)\right) \\
& =1-\sup _{x \in X-B \subseteq X-A} \tau_{\Delta}(X-B) \\
& =1-N_{x}^{\Delta}(X-A) .
\end{aligned}
$$

2) It is similar to the proof of (1).

\section{Corollary 5.3}

1) a) $\bar{A}(x)=1-N_{x}(X-A)$;

b) $C l_{\alpha}(A)(x)=1-N_{x}^{\alpha}(X-A)$;

c) $C l_{S}(A)(x)=1-N_{x}^{S}(X-A)$;

d) $C l_{P}(A)(x)=1-N_{x}^{P}(X-A)$;

e) $C l_{\gamma}(A)(x)=1-N_{x}^{\gamma}(X-A)$;

f) $C l_{\beta}(A)(x)=1-N_{x}^{\beta}(X-A)$;

2) a) $C l_{C \alpha}(A)(x)=1-N_{x}^{C \alpha}(X-A)$;

b) $C l_{C S}(A)(x)=1-N_{x}^{C S}(X-A)$;

c) $C l_{C P}(A)(x)=1-N_{x}^{C P}(X-A)$;

d) $C l_{C \gamma}(A)(x)=1-N_{x}^{C \gamma}(X-A)$;

e) $C l_{C \beta}(A)(x)=1-N_{x}^{C \beta}(X-A)$;

Theorem 5.4 For every $A \subseteq X$ and $x \in X$ we have

1) If $\Delta$ is of type $O 1$, then

a) $\vDash C l_{\Delta}(\phi) \equiv \phi$;

b) $\vDash C l_{C \Delta}(\phi) \equiv \phi$;

2) a) $\vDash A \subseteq C l_{\Delta}(A)$;

b) $\vDash A \subseteq C l_{C \Delta}(A)$;

3) a) $\vDash C l_{\Delta}(A) \equiv d_{\Delta}(A) \cup A$;

b) $\vDash C l_{C \Delta}(A) \equiv d_{C \Delta}(A) \cup A$;

4) a) $\vDash x \in C l_{\Delta}(A) \leftrightarrow \forall B\left(B \in N_{x}^{\Delta} \rightarrow A \cap B \neq \phi\right)$;

b) $\vDash x \in C l_{C \Delta}(A) \leftrightarrow \forall B\left(B \in N_{x}^{C \Delta} \rightarrow A \cap B \neq \phi\right)$;

5) a) $\vDash A \equiv C l_{\Delta}(A) \leftrightarrow A \in \digamma_{\Delta}$;

b) If $\Delta$ is of type $O 1$ and monotone, then

$$
\vDash A \equiv C l_{C \Delta}(A) \leftrightarrow A \in \digamma_{N^{C \Delta}} ;
$$

Proof. We prove only a) of each statements since b) is similar.

1) a) $C l_{\Delta}(\phi)(x)=1-N_{x}(X-\phi)$

$$
=1-N_{x}(X)=1-1=0 .
$$

2) a) It is clear that $N_{x}(A)=0$ for any $A \subseteq X$ and $x \in X$ in case of $x \notin A$. Now, suppose that $x \in A$. Then $C l_{\Delta}(A)(x)=1-N_{x}^{\Delta}(X-A)=1$. Therefore $\vDash A \subseteq C l_{\Delta}(A)$.

3) a) Using Theorem 5.1 (1) and (2) above, we have 
$\left(d_{\Delta}(A) \cup A\right)(x)=\max \left(1-N_{x}^{\Delta}((X-A) \cup\{x\}), A(x)\right)$

$$
=C l_{\Delta}(A)(x) \text {. }
$$

4) a) $\left[\forall B\left(B \in N_{x}^{C \Delta} \rightarrow A \cap B \neq \phi\right)\right]$

$$
\begin{aligned}
& =\inf _{B \subseteq X-A}\left(1-N_{x}^{\Delta}(B)\right)=1-\sup _{B \subseteq X-A} N_{x}^{\Delta}(B) \\
& =1-N_{x}^{\Delta}(X-A)=C l_{\Delta}(A)(x) .
\end{aligned}
$$

5) a) Since $\left[A \subseteq d_{\Delta}(A) \cup A\right]=1$, then from Theorem 5.2 (1) and (3) (a) above we have

$$
\begin{aligned}
\digamma_{\Delta}(A) & =\left[d_{\Delta}(A) \subseteq A\right]=\left[d_{\Delta}(A) \cup A \subseteq A\right] \\
& =\left[A \subseteq d_{\Delta}(A) \cup A\right] \wedge\left[d_{\Delta}(A) \cup A \subseteq A\right] \\
& =\left[d_{\Delta}(A) \cup A \equiv A\right]=\left[C l_{\Delta}(A) \equiv A\right],
\end{aligned}
$$

because $[\tilde{B} \subseteq A]=[\tilde{B} \cup A \equiv A]$ for any $\tilde{B} \in \mathfrak{I}(X)$.

\section{Corollary 5.4}

1) a) $\vDash \bar{\phi} \equiv \phi$; b) $\vDash C l_{\alpha}(\phi) \equiv \phi$; c) $\vDash C l_{S}(\phi) \equiv \phi$; d) $\vDash C l_{P}(\phi) \equiv \phi ;$ e) $\vDash C l_{\gamma}(\phi) \equiv \phi ;$ f) $\left.\vDash C l_{\beta}(\phi) \equiv \phi ; \mathrm{g}\right)$ $\left.\left.\left.\vDash C l_{C \alpha}(\phi) \equiv \phi ; \mathrm{h}\right) \vDash C l_{C S}(\phi) \equiv \phi ; \mathrm{i}\right) \vDash C l_{C P}(\phi) \equiv \phi ; \mathrm{j}\right)$ $\left.\vDash C l_{C \gamma}(\phi) \equiv \phi ; \mathrm{k}\right) \vDash C l_{C \beta}(\phi) \equiv \phi$;

2) for any $A \subseteq X$, a) $\vDash A \subseteq \bar{A}$; b) $\vDash A \subseteq C l_{\alpha}(A)$; c) $\left.\left.\left.\vDash A \subseteq C l_{S}(A) ; \mathrm{d}\right) \vDash A \subseteq C l_{P}(A) ; \mathrm{e}\right) \vDash A \subseteq C l_{\gamma}(A) ; \mathrm{f}\right)$ $\left.\left.\left.\vDash A \subseteq C l_{\beta}(A) ; \mathrm{g}\right) \vDash A \subseteq C l_{C \alpha}(A) ; \mathrm{h}\right) \vDash A \subseteq C l_{C S}(A) ; \mathrm{i}\right)$ $\left.\left.\vDash A \subseteq C l_{C P}(A) ; \mathrm{j}\right) \vDash A \subseteq C l_{C \gamma}(A) ; \mathrm{k}\right) \vDash A \subseteq C l_{C \beta}(A)$.

3) for any $A \subseteq X$, a) $\vDash \bar{A} \equiv A^{\prime} \cup A$; b) $\left.\left.\vDash C l_{\alpha}(A) \equiv d_{\alpha}(A) \cup A ; \mathrm{c}\right) \vDash C l_{S}(A) \equiv d_{S}(A) \cup A ; \mathrm{d}\right)$ $\vDash C l_{P}(A) \equiv d_{P}(A) \cup A ;$ e $\left.) \quad \vDash C l_{\gamma}(A) \equiv d_{\gamma}(A) \cup A ; \mathrm{f}\right)$ $\left.\left.\vDash C l_{\beta}(A) \equiv d_{\beta}(A) \cup A ; \mathrm{g}\right) \vDash C l_{C \alpha}(A) \equiv d_{C \alpha}(A) \cup A ; \mathrm{h}\right)$ $\left.\left.\vDash C l_{C S}(A) \equiv d_{C S}(A) \cup A ; \mathrm{i}\right) \vDash C l_{C P}(A) \equiv d_{C P}(A) \cup A ; \mathrm{j}\right)$ $\left.\vDash C l_{C \gamma}(A) \equiv d_{C \gamma}(A) \cup A ; \mathrm{k}\right) \vDash C l_{C \beta}(A) \equiv d_{C \beta}(A) \cup A$.

4) for any $A \subseteq X$ and $x \in X$,

a) $\vDash \bar{A} \leftrightarrow \forall B\left(B \in N_{x} \rightarrow A \cap B \neq \phi\right)$;

b) $\vDash x \in C l_{\alpha}(A) \leftrightarrow \forall B\left(B \in N_{x}^{\alpha} \rightarrow A \cap B \neq \phi\right)$;

c) $\vDash x \in C l_{S}(A) \leftrightarrow \forall B\left(B \in N_{x}^{S} \rightarrow A \cap B \neq \phi\right)$;

d) $\vDash x \in C l_{P}(A) \leftrightarrow \forall B\left(B \in N_{x}^{P} \rightarrow A \cap B \neq \phi\right)$;

e) $\vDash x \in C l_{\gamma}(A) \leftrightarrow \forall B\left(B \in N_{x}^{\gamma} \rightarrow A \cap B \neq \phi\right)$;

f) $\vDash x \in C l_{\beta}(A) \leftrightarrow \forall B\left(B \in N_{x}^{\beta} \rightarrow A \cap B \neq \phi\right)$;

g) $\vDash x \in C l_{C \alpha}(A) \leftrightarrow \forall B\left(B \in N_{x}^{C \alpha} \rightarrow A \cap B \neq \phi\right)$;

h) $\vDash x \in C l_{C S}(A) \leftrightarrow \forall B\left(B \in N_{x}^{C S} \rightarrow A \cap B \neq \phi\right)$;

i) $\vDash x \in C l_{C P}(A) \leftrightarrow \forall B\left(B \in N_{x}^{C P} \rightarrow A \cap B \neq \phi\right)$; j) $\vDash x \in C l_{C \gamma}(A) \leftrightarrow \forall B\left(B \in N_{x}^{C \gamma} \rightarrow A \cap B \neq \phi\right)$;

k) $\vDash x \in C l_{C \beta}(A) \leftrightarrow \forall B\left(B \in N_{x}^{C \beta} \rightarrow A \cap B \neq \phi\right)$.

5) for any $A \subseteq X$,

a) $\vDash A \equiv \bar{A} \leftrightarrow A \in \digamma$;

b) $\vDash A \equiv C l_{\alpha}(A) \leftrightarrow A \in \digamma_{\alpha}$;

c) $\vDash A \equiv C l_{S}(A) \leftrightarrow A \in \digamma_{S}$;

d) $\vDash A \equiv C l_{P}(A) \leftrightarrow A \in \digamma_{P}$;

e) $\vDash A \equiv C l_{\gamma}(A) \leftrightarrow A \in \digamma_{\gamma}$;

f) $\vDash A \equiv C l_{\beta}(A) \leftrightarrow A \in \digamma_{\beta}$;

g) $\vDash A \equiv C l_{C \alpha}(A) \leftrightarrow A \in \digamma_{N^{C \alpha}}$;

h) $\vDash A \equiv C l_{C S}(A) \leftrightarrow A \in \digamma_{N}$ CS

i) $\vDash A \equiv C l_{C P}(A) \leftrightarrow A \in \digamma_{N^{C P}}$;

j) $\vDash A \equiv C l_{C \gamma}(A) \leftrightarrow A \in \digamma_{N^{C \gamma}}$;

k) $\vDash A \equiv C l_{C \beta}(A) \leftrightarrow A \in \digamma_{N^{C \beta}}$.

Theorem 5.5 For every $A, B \subseteq X$ we have

1) $\vDash B \doteq C l_{\Delta}(A) \leftrightarrow B \in \digamma_{\Delta}$;

2) $\vDash B \doteq C l_{C \Delta}(A) \leftrightarrow B \in \digamma_{N^{C \Delta}}$.

Proof. 1) Suppose $[A \subseteq B]=0$. Then

$\left[B \doteq C l_{\Delta}(A)\right]=0$ and the results holds. Now, suppose $[A \subseteq B]=1$. Then $\left[B \subseteq C l_{\Delta}(A)\right]=1-\sup _{x \in B-A} N_{x}^{\Delta}(X-A)$ and $\left[C l_{\Delta}(A) \subseteq B\right]=\inf _{x \in X-B} N_{x}^{\Delta}(X-A)$. So

$$
\begin{aligned}
& {\left[B \doteq C l_{\Delta}(A)\right]} \\
& =\max \left(0, \inf _{x \in X-B} N_{x}^{\Delta}(X-A)-\sup _{x \in B-A} N_{x}^{\Delta}(X-A)\right) .
\end{aligned}
$$

Let $\left[B \doteq C l_{\Delta}(A)\right]>t$. Then

$\inf _{x \in X-B} N_{x}^{\Delta}(X-A)>t+\sup _{x \in B-A} N_{x}^{\Delta}(X-A)$. For any

$x \in X-B, \quad \sup _{x \in C \subseteq X-A} \tau_{\Delta}(C)>t+\sup _{x \in B-A} N_{x}^{\Delta}(X-A)$, i.e., there exists $C_{x} \subseteq X$ such that $x \in C_{x} \subseteq X-A$ and $\tau_{\Delta}\left(C_{x}\right)>t+\sup _{x \in B-A} N_{x}^{\Delta}(X-A)$. Now we want to prove $C_{x} \subseteq X-B$. If not, then there exist $x^{\prime} \in B-A$ with $x^{\prime} \in C_{x}$. Hence

$$
\begin{aligned}
\sup _{x \in B-A} N_{x}^{\Delta}(X-A) & \geq N_{x^{\prime}}^{\Delta}(X-A) \geq \tau_{\Delta}\left(C_{x}\right) \\
& >t+\sup _{x \in B-A} N_{x}^{\Delta}(X-A)
\end{aligned}
$$

and this is a contradiction. Therefore

$$
\begin{aligned}
\digamma_{\Delta}(B) & =\tau_{\Delta}(X-B)=\inf _{x \in X-B} N_{x}^{\Delta}(X-B) \\
& \geq \inf _{x \in X-B} \tau_{\Delta}\left(C_{x}\right)>t+\sup _{x \in B-A} N_{x}^{\Delta}(X-A)>t .
\end{aligned}
$$


Since $t$ is arbitrary, it holds that $\left[B \doteq C l_{\Delta}(A)\right] \leq \digamma_{\Delta}(B)$

2) The proof is similar to 1 ).

Corollary 5.5 For any $A$ and $B$.

1) a) $\vDash B \doteq \bar{A} \leftrightarrow B \in \digamma$;

b) $\vDash B \doteq C l_{\alpha}(A) \leftrightarrow B \in \digamma_{\alpha}$;

c) $\vDash B \doteq C l_{S}(A) \leftrightarrow B \in \digamma_{S}$;

d) $\vDash B \doteq C l_{P}(A) \leftrightarrow B \in \digamma_{P}$;

e) $\vDash B \doteq C l_{\gamma}(A) \leftrightarrow B \in \digamma_{\gamma}$;

e) $\vDash B \doteq C l_{\beta}(A) \leftrightarrow B \in \digamma_{\beta}$;

2) a) $\vDash B \doteq C l_{C \alpha}(A) \leftrightarrow B \in \digamma_{N^{C \alpha}}$;

b) $\vDash B \doteq C l_{C S}(A) \leftrightarrow B \in \digamma_{N} C S$;

c) $\vDash B \doteq C l_{C P}(A) \leftrightarrow B \in \digamma_{N}{ }^{C P}$;

d) $\vDash B \doteq C l_{C \gamma}(A) \leftrightarrow B \in \digamma_{N^{C \gamma}}$;

e) $\vDash B \doteq C l_{C \beta}(A) \leftrightarrow B \in \digamma_{N}{ }_{N \beta}$.

Definition 5.5 The fuzzifying $\Delta$-(resp. $C \Delta$-) interior Int $_{\Delta}(A)$ (resp. $\left.\operatorname{Int}_{C \Delta}(A)\right) \in \mathfrak{I}(X)$ of $A \subseteq X$ is defined as follows:

$$
x \in \operatorname{Int}_{\Delta}(A):=N_{x}^{\Delta}(A)\left(\operatorname{resp} . x \in \operatorname{Int}_{C \Delta}(A):=N_{x}^{C \Delta}(A)\right) .
$$

Definition 5.6 1) If $\Delta=()^{\circ}$, (resp. ()$^{\circ-\circ},()^{\circ-},()^{-\circ}$, ()$\left.^{-\circ} \cup()^{\circ-},()^{-\circ-}\right)$, the notion of fuzzifying $\Delta$-interior of $A \subseteq X$ coincides with the notion of fuzzifying interior (resp. $\alpha$-interior, semi-interior, pre-interior, $\gamma$-interior, $\beta$-interior) operation and will be denoted by

$A^{\circ}\left(\operatorname{resp} \operatorname{Int}_{\alpha}(A), \operatorname{Int}_{S}(A), \operatorname{Int}_{P}(A), \operatorname{Int}_{\gamma}(A), \operatorname{Int}_{\beta}(A)\right)$;

2) If $\Delta=()^{\circ-\circ}\left(\operatorname{resp} .()^{\circ-},()^{-\circ},()^{-\circ} \cup()^{\circ-},()^{-\circ-}\right)$, the notion of fuzzifying $C \Delta$-interior of $A \subseteq X$ coincides with the notion of fuzzifying $c \alpha$ (resp. csemi, cpre, $c \gamma, c \beta$ )-interior operation and will be denoted by

$\operatorname{Int}_{C \alpha}(A)\left(\operatorname{resp} . \operatorname{Int}_{C S}(A), \operatorname{Int}_{C P}(A), \operatorname{Int}_{C \gamma}(A), \operatorname{Int}_{C \beta}(A)\right)$.

Theorem 5.6 For every $x, A, B$ we have

1) a) $\vDash B \doteq \operatorname{Int}_{\Delta}(A) \rightarrow B \in \tau_{\Delta}$;

b) if $\Delta$ is of type $O 1$ and monotone, then

$$
\vDash B \equiv \operatorname{Int}_{C \Delta}(A) \rightarrow B \in \tau_{N}{ }_{N} \text {; }
$$

2) a) $\vDash B \in \tau_{\Delta} \wedge B \subseteq A \rightarrow B \subseteq \operatorname{Int}_{\Delta}(A)$;

b) if $\Delta$ is of type $\mathrm{O} 1$ and monotone, then

$$
\vDash B \in \tau_{N^{C \Delta}} \wedge B \subseteq A \rightarrow B \subseteq \operatorname{Int}_{\Delta}(A) ;
$$

3) a) $\vDash A \equiv \operatorname{Int}_{\Delta}(A) \leftrightarrow A \in \tau_{\Delta}$;

b) if $\Delta$ is of type $O 1$ and monotone, then

$$
\vDash A \equiv \operatorname{Int}_{C \Delta}(A) \leftrightarrow A \in \tau_{N^{C \Delta}} ;
$$

4) a) $\vDash x \in \operatorname{Int}_{\Delta}(A) \leftrightarrow x \in A \wedge x \in\left(X-d_{\Delta}(X-A)\right)$; b) $\vDash x \in \operatorname{Int}_{C \Delta}(A) \leftrightarrow x \in A \wedge x \in\left(X-d_{C \Delta}(X-A)\right)$;

5) a) $\vDash \operatorname{Int}_{\Delta}(A) \equiv X-C l_{\Delta}(X-A)$;

b) $\vDash \operatorname{Int}_{C \Delta}(A) \equiv X-C l_{C \Delta}(X-A)$;

Proof. We prove only a) of each statements since $b$ ) is similar.

First, we prove 2) a) If $B \nsubseteq A$, then

$\left[B \in \tau_{\Delta} \wedge B \subseteq A\right]=0$. Now, suppose that $B \subseteq A$. Then we have

$$
\begin{aligned}
& {\left[B \subseteq \operatorname{Int}_{\Delta}(A)\right]=\inf _{x \in B} \operatorname{Int}_{\Delta}(A)(x)=\inf _{x \in B} N_{x}^{\Delta}(A)} \\
& \geq \inf _{x \in B} N_{x}^{\Delta}(B)=\tau_{\Delta}(B)=\left[B \in \tau_{\Delta} \wedge B \subseteq A\right] .
\end{aligned}
$$

3) a) $\left[A \equiv \operatorname{Int}_{\Delta}(A)\right]$

$$
\begin{aligned}
& =\min \left(\inf _{x \in A} \operatorname{Int}_{\Delta}(A)(x), \inf _{x \in A}\left(1-\operatorname{Int}_{\Delta}(A)(x)\right)\right) \\
& =\inf _{x \in A} \operatorname{Int}_{\Delta}(A)(x)=\inf _{x \in A} N_{x}^{\Delta}(A) \\
& =\tau_{\Delta}(A)=\left[A \in \tau_{\Delta}\right] .
\end{aligned}
$$

4) a) If $x \notin A$,

$$
\left[x \in \operatorname{Int}_{\Delta}(A)\right]=0=\left[x \in A \wedge x \in\left(X-d_{\Delta}(X-A)\right)\right] \text {. }
$$

If $x \in A$,

$$
\begin{aligned}
{\left[x \in d_{\Delta}(X-A)\right] } & =1-N_{x}^{\Delta}(A \cup\{x\}) \\
& =1-N_{x}^{\Delta}(A)=1-\operatorname{Int}_{\Delta}(A)(x) .
\end{aligned}
$$

Thus $\left[x \in A \wedge x \in\left(X-d_{\Delta}(X-A)\right)\right]=\operatorname{Int}_{\Delta}(A)(x)$.

5) a) Follows from Theorem 5.3 (1).

Finally, we prove 1) a). From 5) a) and Theorem 5.5 1) we have

$$
\begin{aligned}
{\left[B \doteq \operatorname{Int}_{\Delta}(A)\right] } & =\left[X-B \doteq C l_{\Delta}(X-A)\right] \\
& \leq\left[(X-B) \in \digamma_{\Delta}\right]=\tau_{\Delta}(B) .
\end{aligned}
$$

Corollary 5.6 For any $x, A, B$,

1) a) $\vDash B \doteq A^{\circ} \rightarrow B \in \tau$;

b) $\vDash B \doteq \operatorname{Int}_{\alpha}(A) \rightarrow B \in \tau_{\alpha}$;

c) $\vDash B \doteq \operatorname{Int}_{S}(A) \rightarrow B \in \tau_{S}$;

d) $\vDash B \doteq \operatorname{Int}_{P}(A) \rightarrow B \in \tau_{P}$;

e) $\vDash B \doteq \operatorname{Int}_{\gamma}(A) \rightarrow B \in \tau_{\gamma}$;

f) $\vDash B \doteq \operatorname{Int}_{\beta}(A) \rightarrow B \in \tau_{\beta}$;

g) $\vDash B \doteq \operatorname{Int}_{C \alpha}(A) \rightarrow B \in \tau_{N} C \alpha$;

h) $\vDash B \doteq \operatorname{Int}_{C S}(A) \rightarrow B \in \tau_{N^{C S}}$;

i) $\vDash B \doteq \operatorname{Int}_{C P}(A) \rightarrow B \in \tau_{N^{C P}}$;

j) $\vDash B \doteq \operatorname{Int}_{C \gamma}(A) \rightarrow B \in \tau_{N^{C \gamma}}$;

k) $\vDash B \doteq \operatorname{Int}_{C \beta}(A) \rightarrow B \in \tau_{N^{C \beta}}$;

2) a) $\vDash B \in \tau \wedge B \subseteq A \rightarrow B \subseteq A^{\circ}$; 
b) $\vDash B \in \tau_{\alpha} \wedge B \subseteq A \rightarrow B \subseteq \operatorname{Int}_{\alpha}(A)$;

c) $\vDash B \in \tau_{S} \wedge B \subseteq A \rightarrow B \subseteq \operatorname{Int}_{S}(A)$;

d) $\vDash B \in \tau_{P} \wedge B \subseteq A \rightarrow B \subseteq \operatorname{Int}_{P}(A)$;

e) $\vDash B \in \tau_{\gamma} \wedge B \subseteq A \rightarrow B \subseteq \operatorname{Int}_{\gamma}(A)$;

f) $\vDash B \in \tau_{\beta} \wedge B \subseteq A \rightarrow B \subseteq \operatorname{Int}_{\beta}(A)$;

g) $\vDash B \in \tau_{{ }^{C} \alpha} \wedge B \subseteq A \rightarrow B \subseteq \operatorname{Int}_{\alpha}(A)$;

h) $\vDash B \in \tau_{N^{C S}} \wedge B \subseteq A \rightarrow B \subseteq \operatorname{Int}_{S}(A)$;

i) $\vDash B \in \tau_{N^{C P}} \wedge B \subseteq A \rightarrow B \subseteq \operatorname{Int}_{P}(A)$;

j) $\vDash B \in \tau_{N^{c} \gamma} \wedge B \subseteq A \rightarrow B \subseteq \operatorname{Int}_{\gamma}(A)$;

k) $\vDash B \in \tau_{N^{C \beta}} \wedge B \subseteq A \rightarrow B \subseteq \operatorname{Int}_{\beta}(A)$;

3) a) $\vDash A \equiv A^{\circ} \leftrightarrow A \in \tau$;

b) $\vDash A \equiv \operatorname{Int}_{\alpha}(A) \leftrightarrow A \in \tau_{\alpha}$;

c) $\vDash A \equiv \operatorname{Int}_{S}(A) \leftrightarrow A \in \tau_{S}$;

d) $\vDash A \equiv \operatorname{Int}_{P}(A) \leftrightarrow A \in \tau_{P}$;

e) $\vDash A \equiv \operatorname{Int}_{\gamma}(A) \leftrightarrow A \in \tau_{\gamma}$;

f) $\vDash A \equiv \operatorname{Int}_{\beta}(A) \leftrightarrow A \in \tau_{\beta}$;

g) $\vDash A \equiv \operatorname{Int}_{C \alpha}(A) \leftrightarrow A \in \tau_{N^{C \alpha}}$;

h) $\vDash A \equiv \operatorname{Int}_{C S}(A) \leftrightarrow A \in \tau_{N^{C S}}$;

i) $\vDash A \equiv \operatorname{Int}_{C P}(A) \leftrightarrow A \in \tau_{N^{C P}}$;

j) $\vDash A \equiv \operatorname{Int}_{C \gamma}(A) \leftrightarrow A \in \tau_{N^{C \gamma}}$;

k) $\vDash A \equiv \operatorname{Int}_{C \beta}(A) \leftrightarrow A \in \tau_{N^{C \beta}}$;

4) a) $\vDash x \in A^{\circ} \leftrightarrow x \in A \wedge x \in\left(X-(X-A)^{\prime}\right)$;

b) $\vDash x \in \operatorname{Int}_{\alpha}(A) \leftrightarrow x \in A \wedge x \in\left(X-d_{\alpha}(X-A)\right)$;

c) $\vDash x \in \operatorname{Int}_{S}(A) \leftrightarrow x \in A \wedge x \in\left(X-d_{S}(X-A)\right)$;

d) $\vDash x \in \operatorname{Int}_{P}(A) \leftrightarrow x \in A \wedge x \in\left(X-d_{P}(X-A)\right)$;

e) $\vDash x \in \operatorname{Int}_{\gamma}(A) \leftrightarrow x \in A \wedge x \in\left(X-d_{\gamma}(X-A)\right)$;

f) $\vDash x \in \operatorname{Int}_{\beta}(A) \leftrightarrow x \in A \wedge x \in\left(X-d_{\beta}(X-A)\right)$;

g) $\vDash x \in \operatorname{Int}_{C \alpha}(A) \leftrightarrow x \in A \wedge x \in\left(X-d_{C \alpha}(X-A)\right)$;

h) $\vDash x \in \operatorname{Int}_{C S}(A) \leftrightarrow x \in A \wedge x \in\left(X-d_{C S}(X-A)\right)$;

i) $\vDash x \in \operatorname{Int}_{C P}(A) \leftrightarrow x \in A \wedge x \in\left(X-d_{C P}(X-A)\right)$;

j) $\vDash x \in \operatorname{Int}_{C \gamma}(A) \leftrightarrow x \in A \wedge x \in\left(X-d_{C \gamma}(X-A)\right)$;

k) $\vDash x \in \operatorname{Int}_{C \beta}(A) \leftrightarrow x \in A \wedge x \in\left(X-d_{C \beta}(X-A)\right)$;

5) a) $\vDash A^{\circ} \equiv X-\overline{X-A}$;

b) $\vDash \operatorname{Int}_{\alpha}(A) \equiv X-C l_{\alpha}(X-A)$;

c) $\vDash \operatorname{Int}_{S}(A) \equiv X-C l_{S}(X-A)$;

d) $\vDash \operatorname{Int}_{P}(A) \equiv X-C l_{P}(X-A)$; e) $\vDash \operatorname{Int}_{\gamma}(A) \equiv X-C l_{\gamma}(X-A)$;

f) $\vDash \operatorname{Int}_{\beta}(A) \equiv X-C l_{\beta}(X-A)$;

g) $\vDash \operatorname{Int}_{C \alpha}(A) \equiv X-C l_{C \alpha}(X-A)$;

h) $\vDash \operatorname{Int}_{C S}(A) \equiv X-C l_{C S}(X-A)$;

i) $\vDash \operatorname{Int}_{C P}(A) \equiv X-C l_{C P}(X-A)$;

j) $\vDash \operatorname{Int}_{C \gamma}(A) \equiv X-C l_{C \gamma}(X-A)$;

k) $\vDash \operatorname{Int}_{C \beta}(A) \equiv X-C l_{C \beta}(X-A)$.

\section{Conclusions}

The present paper investigates topological notions when these are planted into the framework of Ying's fuzzifying topological spaces (in semantic method of continuous valued-logic). It continue various investigations into fuzzy topology in a legitimate way and extend some fundamental results in general topology to fuzzifying topology. An important virtue of our approach (in which we follow Ying) is that we define topological notions as fuzzy predicates (by formulae of Łukasiewicz fuzzy logic) and prove the validity of fuzzy implications (or equivalences). Unlike the (more wide-spread) style of defining notions in fuzzy mathematics as crisp predicates of fuzzy sets, fuzzy predicates of fuzzy sets provide a more genuine fuzzification; furthermore the theorems in the form of valid fuzzy implications are more general than the corresponding theorems on crisp predicates of fuzzy sets. The main contributions of the paper are to study some sorts of operations, called general fuzzifying operations. There are some problems for further study:

1) Apply the general fuzzifying operation to convergence theory, continuity, separation axioms etc.

2) What is the justification of these concepts in the setting of $(2, L)$ topologies.

\section{REFERENCES}

[1] U. Höhle, "Many Valued Topology and Its Applications," Kluwer Academic Publishers, Dordrecht, 2001. doi:10.1007/978-1-4615-1617-0

[2] U. Höhle and S. E. Rodabaugh, "Mathematics of Fuzzy Sets: Logic, Topology, and Measure Theory," Handbook of Fuzzy Sets Series, Vol. 3, Kluwer Academic Publishers, Dordrecht, 1999.

[3] U. Höhle, S. E. Rodabaugh and A. Ŝstak, "Special Issue on Fuzzy Topology," Fuzzy Sets and Systems, Vol. 73, 1995, pp. 1-183.

[4] T. Kubiak, "On Fuzzy Topologies," Ph.D. Thesis, Adam Mickiewicz University, Poznan, 1985.

[5] Y. M. Liu and M. K. Luo, "Fuzzy Topology," World Scientific, Singapore, 1998.

[6] G. J. Wang, "Theory of L-Fuzzy Topological Spaces," Shaanxi Normal University Press, Xi'an, 1988 (in Chi- 
nese).

[7] C. L. Chang, "Fuzzy Topological Spaces," Journal of Mathematical Analysis and Applications, Vol. 24, No. 1, 1968, pp. 182-190. doi:10.1016/0022-247X(68)90057-7

[8] J. A. Goguen, "The Fuzzy Tychonoff Theorem," Journal of Mathematical Analysis and Applications, Vol. 43, No. 3, 1973, pp. 182-190. doi:10.1016/0022-247X(73)90288-6

[9] J. L. Kelley, "General Topology," Van Nostrand, New York, 1955.

[10] U. Höhle, "Uppersemicontinuous Fuzzy Sets and Applications," Journal of Mathematical Analysis and Applications, Vol. 78, No. 2, 1980, pp. 659-673. doi:10.1016/0022-247X(80)90173-0

[11] U. Höhle and A. Sostak, "Axiomatic Foundations of Fixed-Basis Fuzzy Topology," In: U. Höhle and S. E. Rodabaugh, Eds., Mathematics of Fuzzy Sets: Logic, Topology, and Measure Theory, Vol. 3, Kluwer Academic Publishers, Dordrecht, 1999, pp. 123-272.

[12] S. E. Rodabaugh, "Categorical Foundations of VariableBasis Fuzzy Topology," In: U. Höhle and S. E. Rodabaugh, Eds., Mathematics of Fuzzy Sets: Logic, Topology, and Measure Theory, Vol. 3, Kluwer Academic Publishers, Dordrecht, 1999, pp. 273-388.

[13] J. B. Rosser and A. R. Turquette, "Many-Valued Logics," North-Holland, Amsterdam, 1952.

[14] M. S. Ying, "A New Approach for Fuzzy Topology (I)," Fuzzy Sets and Systems, Vol. 39, No. 3, 1991, pp. 303321. doi:10.1016/0165-0114(91)90100-5

[15] M. S. Ying, "A New Approach for Fuzzy Topology (II)," Fuzzy Sets and Systems, Vol. 47, No. 2, 1992, pp. 221223. doi:10.1016/0165-0114(92)90181-3

[16] K. M. Abd El-Hakeim, F. M. Zeyada and O. R. Sayed, " $\beta$-Continuity and $\mathrm{D}(\mathrm{c}, \beta)$-Continuity in Fuzzifying Topology," The Journal of Fuzzy Mathematics, Vol. 7, No. 3, 1999, pp. 547-558.

[17] K. M. Abd El-Hakeim, F. M. Zeyada and O. R. Sayed,
"Pre-Continuity and D(c, P)-Continuity in Fuzzifying Topology," Fuzzy Sets and Systems, Vol. 119, No. 3, 2001, pp. 459-471. doi:10.1016/S0165-0114(99)00097-4

[18] F. H. Khedr, F. M. Zeyada and O. R. Sayed, "Fuzzy Semi-Continuity and Fuzzy Csemi-Continuity in Fuzzifying Topology," The Journal of Fuzzy Mathematics, Vol. 7, No. 1, 1999, pp. 105-124.

[19] F. H. Khedr, F. M. Zeyada and O. R. Sayed, " $\alpha$-Continuity and $c \alpha$-Continuity in Fuzzifying Topology," Fuzzy Sets and Systems, Vol. 116, No. 3, 2000, pp. 325337. doi:10.1016/S0165-0114(98)00386-8

[20] T. Noiri and O. R. Sayed, "Fuzzy $c \gamma$ Open Sets and Fuzzy $c \gamma$-Continuity in Fuzzifying Topology," International Journal of Mathematics and Mathematical Sciences, Vol. 31, No. 1, 2002, pp. 51-63. doi:10.1155/S0161171202007755

[21] T. Noiri and O. R. Sayed, "Fuzzy $\gamma$ Open Sets and Fuzzy $\gamma$-Continuity in Fuzzifying Topology," Scientiae Mathematicae Japonicae, Vol. 55, No. 2, 2002, pp. 255263.

[22] S. Kasahara, "Operation-Compact Spaces," Mathematica Japonica, 24, No. 1, 1979, pp. 97-105.

[23] D. S. Jankovic', "Properties of $\alpha \beta$-Continuous Functions," The Proceedings of Fifth Prague Topological Symposium, 1981.

[24] M. E. Abd El-Monsef, F. M. Zeyada and A. S. Mashhour, "Operations on Topologies and Its Applications on Some Types of Covering," Annales de la Société Scientifique de Bruxelles, Vol. 79, 1983, pp. 155-172.

[25] E. E. Kerre, A. A. Nouh and A. Kandil, "Operations and the Class of Fuzzy Sets on a Universe Endowed with a Fuzzy Topology," Proceedings of IFSA, Vol. 109-113, Brussels, 1991.

[26] A. Kandil, E. E. Kerre and A. A. Nouh, "Operations and Mappings on Fuzzy Topological Spaces," Annales de la Société Scientifique de Bruxelles, Vol. 105, No. 4, 1991, pp. 167-188. 